ESAIM: M2AN

Vol. 40, No 5, 2006, pp. 871-896

DOI: $10.1051 / \mathrm{m} 2 \mathrm{an}: 2006033$
ESAIM: Mathematical Modelling and Numerical Analysis

www.edpsciences.org/m2an

\title{
A MIXED-FEM AND BEM COUPLING FOR THE APPROXIMATION OF THE SCATTERING OF THERMAL WAVES IN LOCALLY NON-HOMOGENEOUS MEDIA
}

\author{
María-Luisa RAPÚN ${ }^{1}$ AND Francisco-JaVIER SAYAS ${ }^{2}$
}

\begin{abstract}
This paper proposes and analyzes a BEM-FEM scheme to approximate a time-harmonic diffusion problem in the plane with non-constant coefficients in a bounded area. The model is set as a Helmholtz transmission problem with adsorption and with non-constant coefficients in a bounded domain. We reformulate the problem as a four-field system. For the temperature and the heat flux we use piecewise constant functions and lowest order Raviart-Thomas elements associated to a triangulation approximating the bounded domain. For the boundary unknowns we take spaces of periodic splines. We show how to transmit information from the approximate boundary to the exact one in an efficient way and prove well-posedness of the Galerkin method. Error estimates are provided and experimentally corroborated at the end of the work.
\end{abstract}

Mathematics Subject Classification. 65J05, 65N30, 65N38, 65R20.

Received: May 17, 2005. Revised: May 23, 2006.

\section{INTRODUCTION}

In this paper we deal with a diffusion problem arising in the study of the scattering of thermal waves in non-homogeneous media. Mathematically speaking we will be dealing with a Helmholtz transmission problem in the plane with two particular features: (a) the wave number is complex, i.e. there is adsorption; (b) there is a region of the plane where the coefficients of the equation are not constant.

Thermal waves are generated as a consequence of the adsorption of a periodic heating source, created for instance by a modulated laser beam. Photothermal techniques based on the generation and detection of this kind of waves are very powerful tools for non-destructive testing of composite materials. They have been successfully used to detect and characterize structural defects near the surface. For a detailed discussion on thermal waves and their uses we refer to $[1,25,26]$. Some recent papers (see $[10,41]$ and references therein) deal with some models in photothermal science from the point of view of physical experimentation and the search of analytic solutions in some particular cases. Examples of the applicability of techniques based on thermal waves to inverse problems and parameter determination can be found in $[3,16,37]$.

\footnotetext{
Keywords and phrases. Coupling, finite elements, boundary elements, exterior boundary value problem, Helmholtz equation.

${ }^{1}$ Dep. Matemática e Informática, Universidad Pública de Navarra. Campus de Arrosadía, 31006 Pamplona, Spain.

mluisa.rapun@unavarra.es

2 Dep. Matemática Aplicada, Universidad de Zaragoza. C.P.S., 50018 Zaragoza, Spain. jsayas@unizar.es
}

(C) EDP Sciences, SMAI 2007 
This paper deals with materials that have constant thermal conductivity and density in the exterior of a bounded area. Therefore, the exterior domain is governed by a Helmholtz equation with constant complex wave number. Inside the obstacle (the bounded area), coefficients are smooth strictly positive functions.

BEM-FEM coupling procedures are powerful tools for problems where all the difficulties, such as nonhomogeneities of the equations and/or the coefficients, non-linearities, etc., take place in a bounded domain. In the last decades, the coupling of boundary integral and finite element methods has been applied for solving several transmission problems in an unbounded domain with an obstacle in its interior. For this kind of problems, the real interface between the two media can be taken as the coupling boundary as in $[17,29,33]$. Another alternative introduces a new artificial boundary enclosing the obstacle that is used as a coupling boundary. This strategy is commonly used when the interface between the two media is not smooth enough to guarantee well-posedness of the resulting formulation. Some examples of this can be found in $[11,13,27]$. For an exhaustive introduction to these techniques we refer to [12] (see also [7,15] and the pioneering works [5, 20]). Here we will take the physical interface, assumed to be smooth, as coupling boundary. However, when the interface is a polygonal line, it is simple to adapt our method to a smooth interface strictly surrounding the obstacle.

We propose a new four-field formulation for the diffusion problem. As unknowns we take: an exterior density such that the solution is expressed as a single layer potential in the unbounded domain; the trace of the solution in the common interface; the complex amplitude of the thermal wave inside the obstacle and the heat flux inside the obstacle. We thus arrive at a coupled formulation with an elliptic variational form for the interior domain and a system of boundary integral equations for the exterior one. The mixed formulation of the interior problem has two advantages. Firstly, we will have at our hand approximations for both temperature and heat flux. Secondly, the original diffusion equation, where the two physical parameters of the problem (density and conductivity) appear, is transformed into two new ones. In each of them only one of the functions describing the thermal properties of the material appears. This is a more suitable formulation for future studies of related inverse problems.

For the numerical solution we choose a BEM-FEM discretization. We combine a recent technique introduced in [40] that consists of using finite elements on a triangular grid (with spaces of piecewise constant functions for the scalar field and lowest order Raviart-Thomas elements for the flux) with a conforming approximation of the boundary unknowns. These unknowns on the boundary are previously transformed into 1-periodic functions by using a smooth parameterization of the boundary. We choose spaces of periodic piecewise constant functions and continuous piecewise linear functions defined on uniform staggered grids. This parameterized version of the boundary unknowns endeavours to approximate easily the weakly singular boundary integrals by quadrature rules (see $[27,31,35,40])$. The aim of this choice is to avoid the main drawbacks of the coupling strategies. On the one hand, we use traditional finite element spaces instead of elements defined on curved triangles which are rarely employed in standard FEM packages. On the other hand, the conforming discretization on the boundary preserves the good properties of the integral operators, which would be lost by approximating the interface by the boundary of the polygonal domain. At this stage, the only critical point is how to transfer information from the interior to the boundary. We solve this problem by copying degrees of freedom in a very straightforward way.

We point out that the discretization parameters for the interior and the exterior domains will be completely unrelated. Matching the boundary gridpoints with the nodes of the triangulation lying on it simplifies the numerical method (from the point of view of implementation) but it is by no means necessary.

The analysis of the scheme is carried out by comparing it with an auxiliary BEM-FEM method where the finite elements are defined over the induced curved triangulations. We follow here some ideas of [40].

There is some previous work having common features with this paper. A related formulation, with the same idea of using a copy of the interior trace as additional unknown, appears in $[32,35]$ applied to a problem of fluid-solid interaction. The method presented there uses ideal curved triangles and spectral approximation of the boundary unknowns. Form the point of view of the mixed formulation of the interior problem and the choice of the discrete spaces we find some similarities in [34] for an exterior Dirichlet problem for the Laplace equation. 
Although the FEM discretization is defined on a polygonal domain approximating the original one, the spline approximation of the boundary unknowns is carried out on the boundary of the polygonal domain.

Our method has several advantages for this particular type of problems. It is very simple from the point of view of implementation. The BEM and FEM routines work independently and the only coupling term requires a simple location of the exterior nodes of the triangulation with respect to the boundary grid. We work with very elementary finite and boundary elements and are therefore limited to a first order method. There are several possibilities to increase order: (a) use isoparametric elements in the interior and increase the degree of the splines on the boundary; (b) use ideal triangles for the interior, allowing higher order approximations and increase also the order of the boundary approximations (even a spectral method based on trigonometric polynomials can be used). Although the ideal triangles option may seen involved, the work in [30-32,35] points out how to deal with the numerical quadrature issues stemming from the use of ideal triangles. Let us also point out that this low order method poses a challenge on the analytical part of the question, which we solve by a simple and novel (to the best of our knowledge) adaptation of the Strang variational crime theory.

The paper is organized as follows. In the first part, consisting of Sections 1 and 2 we describe the problem, give its BEM-FEM formulation and show its well-possedness. In Section 3 we propose the numerical method, which is analyzed in Sections 5, 6, 7 and 8 using the abstract results of Section 4. Finally, in Section 9 we present some numerical experiments.

Notation. Throughout this work, $C$ (also $C^{\prime}, C^{\prime \prime}, C_{1}, C_{2}$, etc.) denotes a positive constant independent of both discretization parameters $h$ and $H$ and of any quantity it is multiplied by, being possibly different in each occurrence.

All brackets $(\cdot, \cdot),\langle\cdot, \cdot\rangle, \ldots$, will be sesquilinear (linear in the left component and conjugate linear in the second one) both for inner products and (anti)duality products.

\section{Modelling}

Let $\Omega_{-} \subset \mathbb{R}^{2}$ be a simply connected Lipschitz open set and $\Omega_{+}:=\mathbb{R}^{2} \backslash \bar{\Omega}_{-}$its complementary set. The material occupying $\Omega_{+}$is assumed to be homogeneous whereas the bounded domain is occupied by a heterogeneous one. Hence, the conductivity $\sigma: \mathbb{R}^{2} \rightarrow \mathbb{C}$ as well as the density $\rho: \mathbb{R}^{2} \rightarrow \mathbb{C}$ (multiplied by specific heat capacity), will satisfy the following properties:

$$
\begin{aligned}
& \sigma \equiv \sigma_{0}>0, \quad \text { in } \Omega_{+}, \quad \sigma(\cdot) \geq \sigma_{1}>0, \quad \text { in } \Omega_{-}, \\
& \rho \equiv \rho_{0}>0, \quad \text { in } \Omega_{+}, \quad \rho(\cdot) \geq \rho_{1}>0, \quad \text { in } \Omega_{-} .
\end{aligned}
$$

For simplicity we will require that $\sigma \in W^{1, \infty}\left(\Omega_{-}\right)$. The forthcoming analysis is still valid for piecewise smooth $\left.\sigma\right|_{\Omega_{-}}$if the discretization respects the discontinuities of the material coefficients. We also impose that $\rho \in$ $L^{\infty}\left(\Omega_{-}\right)$.

We look for solutions to the diffusion problem

$$
-\nabla \cdot(\sigma \nabla U)+\rho \partial_{t} U=0
$$

that are time-harmonic, i.e. functions $U: \mathbb{R}^{2} \times \mathbb{R} \rightarrow \mathbb{C}$ of the form $U(\mathbf{z}, t)=\operatorname{Re}\left(\mathrm{e}^{-\imath \omega t} v(\mathbf{z})\right)$. Thus, the complex amplitude of the thermal wave $v$ has to be a solution to

$$
\nabla \cdot(\sigma \nabla v)+\imath \omega \rho v=0, \quad \text { in } \mathbb{R}^{2} .
$$


We impose continuity of $v$ and of the heat flux through any discontinuity of the coefficients. We just write explicitly the conditions on $\Gamma:=\partial \Omega_{-}$, since those in other interfaces would be imposed in the variational formulation:

$$
\begin{aligned}
\left.v\right|_{\Gamma} ^{+} & =\left.v\right|_{\Gamma} ^{-}, \\
\left.\sigma_{0} \partial_{\nu} v\right|_{\Gamma} ^{+} & =\left.\sigma \partial_{\nu} v\right|_{\Gamma} ^{-} .
\end{aligned}
$$

We also impose that $v=u_{\text {inc }}+u_{\text {scat }}$ in $\Omega_{+}$, where $u_{\text {inc }}$ (which plays the role of an incident wave) is a known solution to

$$
\nabla \cdot\left(\sigma_{0} \nabla u_{\text {inc }}\right)+\imath \omega \rho_{0} u_{\text {inc }}=0, \quad \text { in } \mathcal{O}
$$

$\mathcal{O}$ being an open set strictly containing $\bar{\Omega}_{-}$, or equivalently, to the Helmholtz equation with adsorption

$$
\Delta u_{\text {inc }}+\lambda^{2} u_{\text {inc }}=0, \quad \text { in } \mathcal{O},
$$

with $\lambda:=(1+\imath) \sqrt{\frac{\rho_{0} \omega}{2 \sigma_{0}}}$. Also, $u_{\text {scat }}$ has to decay at infinity in a way we will shortly specify.

For simplicity, we change notations taking as new unknown

$$
u:=\mid \begin{array}{ll}
v, & \text { in } \Omega_{-}, \\
u_{\text {scat }}, & \text { in } \Omega_{+} .
\end{array}
$$

Defining $g_{0}:=\left.u_{\text {inc }}\right|_{\Gamma} ^{+}$and $g_{1}:=\left.\sigma_{0} \partial_{\nu} u_{\text {inc }}\right|_{\Gamma} ^{+}$, we arrive at the following set of equations

$$
\mid \begin{array}{ll}
u \in H^{1}\left(\Omega_{-} \cup \Omega_{+}\right), & \\
\Delta u+\lambda^{2} u=0, & \text { in } \Omega_{+}, \\
\nabla \cdot(\sigma \nabla u)+\imath \omega \rho u=0, & \text { in } \Omega_{-}, \\
\left.u\right|_{\Gamma} ^{-}-\left.u\right|_{\Gamma} ^{+}=g_{0}, & \\
\left.\sigma \partial_{\nu} u\right|_{\Gamma} ^{-}-\left.\sigma_{0} \partial_{\nu} u\right|_{\Gamma} ^{+}=g_{1} . &
\end{array}
$$

Notice that for exterior Helmholtz problems, it is common to impose the Sommerfeld radiation condition at infinity (see $[6])$. Since we have adsorption ( $\lambda$ has a non-zero imaginary part), this condition is not necessary and can be substituted by the demand that the exterior solution belongs to $H^{1}\left(\Omega_{+}\right)$.

Notice that if

$$
\sigma(\cdot) \equiv \sigma_{1}, \quad \rho(\cdot) \equiv \rho_{1}, \quad \text { in } \Omega_{-},
$$

the problem above is just a transmission problem for the Helmholtz equation with adsorption. Helmholtz transmission problems have been widely studied in the last decades (see for instance $[8,21,23,39,42,43]$ ), with an emphasis in the so-called acoustic case, that is, the case when $\lambda \in \mathbb{R}$. In our context, since $\sigma$ and $\rho$ satisfy the conditions (1.1), uniqueness of solution to problem (1.2) in the homogeneous case (that is, with $g_{0}=g_{1}=0$ ) can be proven by straightforward adaptations of [23], Theorem 3.1. Therefore problem (1.2) has at most one solution.

\section{Formulation}

At this stage, let us assume that $\Gamma$ is a Lyapunov curve (see [36]). For the numerical method we will add some smoothness assumptions on $\Gamma$. We consider the single layer potential

$$
\mathcal{S}_{\lambda} \psi:=\frac{\imath}{4} \int_{\Gamma} H_{0}^{(1)}(\lambda|\cdot-\mathbf{y}|) \psi(\mathbf{y}) \mathrm{d} \gamma_{\mathbf{y}}: \mathbb{R}^{2} \rightarrow \mathbb{C}
$$


where $H_{0}^{(1)}$ is the Hankel function of the first kind and order zero. We define the classical boundary integral operators

$$
\begin{aligned}
V_{\lambda} \psi & :=\frac{\imath}{4} \int_{\Gamma} H_{0}^{(1)}(\lambda|\cdot-\mathbf{y}|) \psi(\mathbf{y}) \mathrm{d} \gamma_{\mathbf{y}}: \Gamma \rightarrow \mathbb{C}, \\
J_{\lambda} \psi & :=\frac{\imath}{4} \int_{\Gamma} \partial_{\nu(\cdot)} H_{0}^{(1)}(\lambda|\cdot-\mathbf{y}|) \psi(\mathbf{y}) \mathrm{d} \gamma_{\mathbf{y}}: \Gamma \rightarrow \mathbb{C} .
\end{aligned}
$$

It is well known (see for instance [8] or [6], Chap. 7) that the single layer potential satisfies

$$
\left.\mathcal{S}_{\lambda} \psi\right|_{\Gamma} ^{+}=V_{\lambda} \psi,\left.\quad \partial_{\nu}\left(\mathcal{S}_{\lambda} \psi\right)\right|_{\Gamma} ^{+}=-\frac{1}{2} \psi+J_{\lambda} \psi
$$

Also (see [28]),

$$
\operatorname{Re}\left[(1-\imath)\left\langle V_{\lambda} \psi, \psi\right\rangle\right] \geq C_{\lambda}\|\psi\|_{-1 / 2, \Gamma}^{2}, \quad \forall \psi \in H^{-1 / 2}(\Gamma),
$$

where $\langle\cdot, \cdot\rangle$ is the antiduality product $H^{ \pm 1 / 2}(\Gamma) \times H^{\mp 1 / 2}(\Gamma)$.

Four unknowns are taken into account: an exterior density $\psi \in H^{-1 / 2}(\Gamma)$ such that $u=\mathcal{S}_{\lambda} \psi$ in $\Omega_{+}$, the interior trace of the solution $\xi=\left.u\right|_{\Gamma} ^{-} \in H^{1 / 2}(\Gamma)$, and a couple of unknowns defined in the interior domain, the amplitude itself $u_{-} \in L^{2}\left(\Omega_{-}\right)$and the heat flux $\mathbf{p}=\sigma \nabla u_{-} \in H\left(\operatorname{div}, \Omega_{-}\right):=\left\{\mathbf{q} \in\left(L^{2}\left(\Omega_{-}\right)\right)^{2} \mid \operatorname{div} \mathbf{q} \in L^{2}\left(\Omega_{-}\right)\right\}$ (see [4]). In differential form we consider the problem

$$
\mid \begin{array}{ll}
(1 / \sigma) \mathbf{p}-\nabla u_{-}=0, & \text { in } \Omega_{-}, \\
\nabla \cdot \mathbf{p}+\imath \omega \rho u_{-}=0, & \text { in } \Omega_{-}, \\
\left.u_{-}\right|_{\Gamma}-\xi=0, & \text { on } \Gamma, \\
\xi-V_{\lambda} \psi=g_{0}, & \text { on } \Gamma, \\
\mathbf{p} \cdot \nu+\sigma_{0}\left(\frac{1}{2} \psi-J_{\lambda} \psi\right)=g_{1}, & \text { on } \Gamma .
\end{array}
$$

If we have a solution to problem (2.2), then the pair $\left(u_{-}, S_{\lambda} \psi\right)$ solves problem (1.2). Moreover, as $\lambda^{2} \notin \mathbb{R}_{+}$it follows that $u_{+}=S_{\lambda} \psi$ belongs to $H^{1}\left(\Omega_{+}\right)$.

We consider the normal trace operator $\gamma: H\left(\operatorname{div}, \Omega_{-}\right) \rightarrow H^{-1 / 2}(\Gamma)$ given by $\gamma \mathbf{p}:=\mathbf{p} \cdot \nu$. This definition has to be understood as the linear and continuous extension of the same operator defined in $\mathcal{C}^{\infty}\left(\bar{\Omega}_{-}\right)^{2}$ or also in a variational setting, using the divergence theorem as definition (see [14]). We also introduce the sesquilinear forms

$$
\begin{array}{ll}
a: H\left(\operatorname{div}, \Omega_{-}\right) \times H\left(\operatorname{div}, \Omega_{-}\right) \longrightarrow \mathbb{C}, & a(\mathbf{p}, \mathbf{q}):=\int_{\Omega_{-}}(1 / \sigma) \mathbf{p} \cdot \overline{\mathbf{q}} \\
b: H\left(\operatorname{div}, \Omega_{-}\right) \times L^{2}\left(\Omega_{-}\right) \longrightarrow \mathbb{C}, & b(\mathbf{p}, v):=\int_{\Omega_{-}}(\nabla \cdot \mathbf{p}) \bar{v}=b^{*}(\bar{v}, \overline{\mathbf{p}}) .
\end{array}
$$

Denoting the inner product in $L^{2}\left(\Omega_{-}\right)$by $(\cdot, \cdot)$, we obtain the variational problem

$$
\mid \begin{array}{ll}
\mathbf{p} \in H\left(\operatorname{div}, \Omega_{-}\right), u \in L^{2}\left(\Omega_{-}\right), \xi \in H^{1 / 2}(\Gamma), \psi \in H^{-1 / 2}(\Gamma), & \\
a(\mathbf{p}, \mathbf{q})+b^{*}(u, \mathbf{q})-\langle\xi, \gamma \mathbf{q}\rangle=0, & \forall \mathbf{q} \in H\left(\operatorname{div}, \Omega_{-}\right), \\
b(\mathbf{p}, v)+\imath \omega(\rho u, v)=0, & \forall v \in L^{2}\left(\Omega_{-}\right), \\
\langle\gamma \mathbf{p}, \eta\rangle+\sigma_{0}\left\langle\frac{1}{2} \psi-J_{\lambda} \psi, \eta\right\rangle=\left\langle g_{1}, \eta\right\rangle, & \forall \eta \in H^{1 / 2}(\Gamma), \\
\quad-\langle\xi, \varphi\rangle+\left\langle V_{\lambda} \psi, \varphi\right\rangle=-\left\langle g_{0}, \varphi\right\rangle, & \forall \varphi \in H^{-1 / 2}(\Gamma) .
\end{array}
$$


To prove well-posedness of problem (2.3), we will transform it into an equivalent operator equation. We write $H\left(\operatorname{div}, \Omega_{-}\right)^{*}$ for the antidual space of $H\left(\operatorname{div}, \Omega_{-}\right)$and $(\cdot, \cdot)$ div for the antiduality product. We could use here the Riesz-Fréchet identification of $H\left(\operatorname{div}, \Omega_{-}\right)^{*}$ and $H\left(\operatorname{div}, \Omega_{-}\right)$, in which case $(\cdot, \cdot)$ div is just the inner product. This is totally immaterial for what follows.

We consider the operator $A: H\left(\operatorname{div}, \Omega_{-}\right) \rightarrow H\left(\operatorname{div}, \Omega_{-}\right)^{*}$ given by

$$
(A \mathbf{p}, \mathbf{q})_{\operatorname{div}}=a(\mathbf{p}, \mathbf{q}), \quad \forall \mathbf{p}, \mathbf{q} \in H\left(\operatorname{div}, \Omega_{-}\right) .
$$

The operator $A$ is one-to-one but $\mathcal{R}(A)$ is not closed. We likewise define $B: H\left(\operatorname{div}, \Omega_{-}\right) \rightarrow L^{2}\left(\Omega_{-}\right) \cong L^{2}\left(\Omega_{-}\right)^{*}$ by

$$
(B \mathbf{p}, v)=b(\mathbf{p}, v), \quad \forall \mathbf{p}, \in H\left(\operatorname{div}, \Omega_{-}\right), \quad v \in L^{2}\left(\Omega_{-}\right) .
$$

It is clear that $B \mathbf{p}=\nabla \cdot \mathbf{p}$. We also introduce the operators $B^{*}: L^{2}\left(\Omega_{-}\right) \rightarrow H\left(\operatorname{div}, \Omega_{-}\right)^{*}$ and $\gamma^{*}: H^{1 / 2}(\Gamma) \rightarrow$ $H\left(\operatorname{div}, \Omega_{-}\right)^{*}$ given by

$$
\begin{array}{ll}
\left(\mathbf{p}, B^{*} u\right)_{\operatorname{div}}=(B \mathbf{p}, u), & \forall u \in L^{2}\left(\Omega_{-}\right), \quad \mathbf{p} \in H\left(\operatorname{div}, \Omega_{-}\right), \\
\left(\mathbf{p}, \gamma^{*} \eta\right)_{\operatorname{div}}=\langle\gamma \mathbf{p}, \eta\rangle, & \forall \eta \in H^{1 / 2}(\Gamma), \quad \mathbf{p} \in H\left(\operatorname{div}, \Omega_{-}\right) .
\end{array}
$$

With a slight abuse of notation we write the multiplication operator $\rho: L^{2}\left(\Omega_{-}\right) \rightarrow L^{2}\left(\Omega_{-}\right)$that maps $u \mapsto \rho u$. With these operators, we construct the operator

$$
\begin{aligned}
\mathcal{A}: \quad H\left(\operatorname{div}, \Omega_{-}\right) & \times L^{2}\left(\Omega_{-}\right) \times H^{1 / 2}(\Gamma) \times H^{-1 / 2}(\Gamma) \\
& \longrightarrow H\left(\operatorname{div}, \Omega_{-}\right)^{*} \times L^{2}\left(\Omega_{-}\right) \times H^{-1 / 2}(\Gamma) \times H^{1 / 2}(\Gamma)
\end{aligned}
$$

given by

$$
\mathcal{A}:=\left[\begin{array}{cccc}
A & B^{*} & -\gamma^{*} & 0 \\
B & \imath \omega \rho & 0 & 0 \\
\gamma & 0 & 0 & \sigma_{0}\left(\frac{1}{2} I-J_{\lambda}\right) \\
0 & 0 & -I & V_{\lambda}
\end{array}\right] .
$$

If we denote $\mathcal{V}:=H\left(\operatorname{div}, \Omega_{-}\right) \times L^{2}\left(\Omega_{-}\right) \times H^{1 / 2}(\Gamma) \times H^{-1 / 2}(\Gamma)$, then we can write the variational problem $(2.3)$ as

$$
\mid \begin{aligned}
& \boldsymbol{\varphi}:=(\mathbf{p}, u, \xi, \psi) \in \mathcal{V}, \\
& \langle\mathcal{A} \boldsymbol{\varphi}, \boldsymbol{\psi}\rangle=\langle g, \boldsymbol{\psi}\rangle, \quad \forall \boldsymbol{\psi} \in \mathcal{V} .
\end{aligned}
$$

Now $\langle\cdot, \cdot\rangle$ denotes the product $\mathcal{V}^{*} \times \mathcal{V}$ and $g:=\left(0,0, g_{1},-g_{0}\right)^{\top}$. Obviously, $\varphi$ is a solution to problem (2.3) if and only if $\mathcal{A} \varphi=g$.

Proposition 2.1. The operator $\mathcal{A}$ is Fredholm of index zero.

Proof. Recall that if $\Gamma$ is Lyapunov, then $J_{\lambda}: H^{-1 / 2}(\Gamma) \rightarrow H^{-1 / 2}(\Gamma)$ is compact. Let $\mathcal{A}_{0}$ be given by

$$
\mathcal{A}_{0}:=\left[\begin{array}{cccc}
A & B^{*} & -\gamma^{*} & 0 \\
B & \imath \omega \rho & 0 & 0 \\
\gamma & 0 & 0 & \frac{\sigma_{0}}{2} I \\
0 & 0 & -I & V_{\lambda}
\end{array}\right],
$$


and let us remark that $\mathcal{A}-\mathcal{A}_{0}$ is compact. It is straightforward to check that $\mathcal{A}_{0}$ can be decomposed as

$$
\left[\begin{array}{cccc}
I & -\imath B^{*}\left(\frac{1}{\omega \rho}\right) & -2 \gamma^{*} V_{\lambda} & 0 \\
0 & I & 0 & 0 \\
0 & 0 & I & -V_{\lambda}^{-1} \\
0 & 0 & 0 & \frac{2}{\sigma_{0}} I
\end{array}\right]\left[\begin{array}{cccc}
Q & 0 & 0 & 0 \\
0 & \imath \omega \rho & 0 & 0 \\
0 & 0 & V_{\lambda}^{-1} & 0 \\
0 & 0 & 0 & V_{\lambda}
\end{array}\right]\left[\begin{array}{cccc}
I & 0 & 0 & 0 \\
-\imath\left(\frac{1}{\omega \rho}\right) B & I & 0 & 0 \\
V_{\lambda} \gamma & 0 & \frac{1}{2} I & 0 \\
0 & 0 & \frac{\sigma_{0}}{2} V_{\lambda}^{-1} & -\frac{\sigma_{0}}{2} I
\end{array}\right]
$$

with

We remark that

$$
Q:=A+\imath B^{*}\left(\frac{1}{\omega \rho}\right) B+2 \gamma^{*} V_{\lambda} \gamma
$$

$$
\operatorname{Re}\left[(1-\imath)(A \mathbf{p}, \mathbf{p})_{\operatorname{div}}\right]=\int_{\Omega_{-}}(1 / \sigma)|\mathbf{p}|^{2}
$$

that

$$
\operatorname{Re}\left[(1-\imath)\left(\imath B^{*}\left(\frac{1}{\omega \rho}\right) B \mathbf{p}, \mathbf{p}\right)_{\operatorname{div}}\right]=\operatorname{Re}\left[(1+\imath)\left(\frac{1}{\omega \rho} B \mathbf{p}, B \mathbf{p}\right)\right]=\int_{\Omega_{-}} \frac{1}{\omega \rho}|\nabla \cdot \mathbf{p}|^{2},
$$

and finally that

$$
\operatorname{Re}\left[(1-\imath)\left(\gamma_{\nu}^{*} V_{\lambda} \gamma_{\nu} \mathbf{p}, \mathbf{p}\right)_{\operatorname{div}}\right]=\operatorname{Re}\left[(1-\imath)\left\langle V_{\lambda} \gamma_{\nu} \mathbf{p}, \gamma_{\nu} \mathbf{p}\right\rangle\right] \geq 0
$$

by inequality (2.1). The hypotheses on $\sigma$ and $\rho$ guarantee then that $Q: H\left(\operatorname{div}, \Omega_{-}\right) \rightarrow H\left(\operatorname{div}, \Omega_{-}\right)^{*}$ satisfies

$$
\operatorname{Re}\left[(1-\imath)(Q \mathbf{p}, \mathbf{p})_{\operatorname{div}}\right] \geq \alpha\|\mathbf{p}\|_{\text {div }}^{2}, \quad \forall \mathbf{p} \in H\left(\operatorname{div}, \Omega_{-}\right)
$$

Therefore $\mathcal{A}_{0}$ is invertible and $\mathcal{A}=\mathcal{A}_{0}+\left(\mathcal{A}-\mathcal{A}_{0}\right)$ is Fredholm of index zero.

Proposition 2.2. The operator $\mathcal{A}$ is an isomorphism.

Proof. By Proposition 2.1, we just have to prove that $\mathcal{A}$ is one-to-one. If $\mathcal{A}(\mathbf{p}, u, \xi, \psi)=0$, then $\mathbf{p}=\sigma \nabla u$ and therefore $u \in H^{1}\left(\Omega_{-}\right)$. Then we prove that

$$
\left.u\right|_{\Gamma}=\xi, \quad \nabla \cdot(\sigma \nabla u)+\imath \omega \rho u=0
$$

and that the couple $\left(u, \mathcal{S}_{\lambda} \psi\right)$ is a solution to the homogeneous transmission problem, i.e. to problem (1.2) with $g_{0}=g_{1}=0$. By uniqueness of solution to this problem (see the end of Sect. 1 ), it follows now that $u=0$ and $\psi=0$, since taking the exterior trace, $V_{\lambda} \psi=0$ and $V_{\lambda}$ is invertible. Therefore $\mathbf{p}=\mathbf{0}$ and $\xi=0$.

Notice that Proposition 2.2 proves existence of solution of problem (1.2) and well-posedness of the variational problem (2.3). This same result also works in the three-dimensional case. The only requirement on $\Gamma$ is Lyapunov regularity, which we will increase to smoothness for numerical analysis. We just have to change the fundamental solution for the Helmholtz equation in potentials and operators. It is also simple to see that the formulation can be extended to non-connected obstacles, i.e. to the situation where $\Gamma$ is a collection of Lyapunov boundaries with non-intersecting interiors.

If the boundary can be parameterized, it is possible to give an equivalent form using periodic Sobolev spaces instead of Sobolev spaces on the boundary $\Gamma$. Since it will be this last formulation the one we will use for numerical approximation, let us briefly write down all the operators in their parameterized form. In general, for $s \in \mathbb{R}$, we define the periodic Sobolev space

$$
H^{s}:=\left\{\left.\varphi \in \mathcal{D}^{\prime}|| \widehat{\varphi}(0)\right|^{2}+\sum_{0 \neq z \in \mathbb{Z}}|k|^{2 s}|\widehat{\varphi}(k)|^{2}<\infty\right\}
$$


where $\mathcal{D}^{\prime}$ is the space of 1-periodic distributions on the real line and $\widehat{\varphi}(k)$ are the Fourier coefficients of $\varphi$. A detailed description of these spaces and their properties can be found in [22], Chapter 8 .

We will assume henceforth that $\Gamma$ is a $\mathcal{C}^{\infty}$ curve that can be described by a smooth regular 1-periodic parameterization, $\mathbf{x}: \mathbb{R} \rightarrow \Gamma$, satisfying

$$
\left|\mathbf{x}^{\prime}(\cdot)\right|>0, \quad \mathbf{x}(t) \neq \mathbf{x}(s), \quad s-t \notin \mathbb{Z} .
$$

This smoothness requirement can be relaxed, but we will assume maximum regularity for the sake of simplicity.

We keep the same notation for the potential

$$
\mathcal{S}_{\lambda} \psi:=\frac{\imath}{4} \int_{0}^{1} H_{0}^{(1)}(\lambda|\cdot-\mathbf{x}(t)|) \psi(t) \mathrm{d} t: \mathbb{R}^{2} \rightarrow \mathbb{C},
$$

and its related operators

$$
\begin{aligned}
V_{\lambda} \psi & :=\frac{\imath}{4} \int_{0}^{1} H_{0}^{(1)}(\lambda|\mathbf{x}(\cdot)-\mathbf{x}(t)|) \psi(t) \mathrm{d} t: \mathbb{R} \rightarrow \mathbb{C} \\
J_{\lambda} \psi & :=\frac{\imath}{4} \int_{0}^{1}\left|\mathbf{x}^{\prime}(\cdot)\right| \partial_{\nu(\mathbf{x}(\cdot))} H_{0}^{(1)}(\lambda|\mathbf{x}(\cdot)-\mathbf{x}(t)|) \psi(t) \mathrm{d} t: \mathbb{R} \rightarrow \mathbb{C}
\end{aligned}
$$

In this case, the jump relations read

$$
\left.\mathcal{S}_{\lambda} \psi\right|_{\Gamma} ^{+} \circ \mathbf{x}=V_{\lambda} \psi,\left.\quad\left|\mathbf{x}^{\prime}\right| \partial_{\nu} \mathcal{S}_{\lambda} \psi\right|_{\Gamma} ^{+} \circ \mathbf{x}=-\frac{1}{2} \psi+J_{\lambda} \psi .
$$

We also need a parameterized form of the normal trace $\gamma: H\left(\operatorname{div}, \Omega_{-}\right) \rightarrow H^{-1 / 2}$ corresponding (formally) to $\gamma_{\nu} \mathbf{p}:=\left|\mathbf{x}^{\prime}\right|(\mathbf{p} \cdot \nu) \circ \mathbf{x}$. We also write $\langle\cdot, \cdot\rangle$ for the antiduality product between $H^{ \pm 1 / 2}$ and $H^{\mp 1 / 2}$, which extends the $H^{0}$ product, and take

$$
g_{0}:=u_{\text {inc }} \circ \mathbf{x}, \quad g_{1}:=\left|\mathbf{x}^{\prime}\right| \sigma_{0} \partial_{\nu} u_{\text {inc }} \circ \mathbf{x},
$$

as new functions for the right-hand side. The modified variational form with this parameterized boundary is simply problem (2.3), changing the spaces $H^{ \pm 1 / 2}(\Gamma)$ by $H^{ \pm 1 / 2}$. We will refer to the variational problem (2.3) understanding that this substitution has been performed. Notice that the boundary unknowns have to be modified as follows in the parameterized case:

$$
\xi \longleftrightarrow \xi \circ \mathbf{x}, \quad \psi \longleftrightarrow\left|\mathbf{x}^{\prime}\right| \psi \circ \mathbf{x} .
$$

\section{Discretization}

We begin by considering the geometric aspects of the discretization: $\Omega_{h}$ is a polygonal approximation of $\Omega_{-}$stemming from a triangulation $\mathcal{T}_{h}$ formed by non-degenerate shape-regular triangles. The parameter to discretize the unit interval is $H:=1 / N$, and we take a couple of staggered grids

$$
s_{j}:=j H, \quad t_{j}:=(j-1 / 2) H, \quad j \in \mathbb{Z} .
$$

We could make the points $\mathbf{x}\left(t_{j}\right)$ (or the points $\mathbf{x}\left(s_{j}\right)$ ) coincide with the vertices of the interior triangulation lying on $\Gamma$. This coincidence simplifies some aspects of the numerical approximation. Nevertheless, we will keep a more general setting, by locating the parameters $\left\{z_{j}\right\}$ such that $\left\{\mathbf{x}\left(z_{j}\right)\right\}$ are the exterior vertices of $\mathcal{T}_{h}$.

The discrete spaces we choose are rather simple:

- $X_{h} \subset H\left(\operatorname{div}, \Omega_{h}\right)$ is the lowest order Raviart-Thomas $\left(\mathcal{R} \mathcal{T}_{0}\right)$ space associated to the triangulation (see [4]).

- $L_{h} \subset L^{2}\left(\Omega_{h}\right)$ is formed by piecewise constant functions on the triangulation. 
- $S_{H} \subset H^{1 / 2}$ is the space of periodic continuous piecewise linear functions with knots on $\left\{s_{j}\right\}$.

- $T_{H} \subset H^{-1 / 2}$ is the space of periodic piecewise constant functions with breakpoints on $\left\{t_{j}\right\}$.

This choice enforces the definition of approximate sesquilinear forms $a_{h} \approx a$ and $b_{h} \approx b$ :

$$
\begin{array}{ll}
a_{h}: X_{h} \times X_{h} \longrightarrow \mathbb{C}, & a_{h}\left(\mathbf{p}_{h}, \mathbf{q}_{h}\right):=\int_{\Omega_{h}}(1 / \sigma) \mathbf{p}_{h} \cdot \overline{\mathbf{q}}_{h}, \\
b_{h}: X_{h} \times L_{h} \longrightarrow \mathbb{C}, & b_{h}\left(\mathbf{p}_{h}, v_{h}\right):=\int_{\Omega_{h}}\left(\nabla \cdot \mathbf{p}_{h}\right) \bar{v}_{h} .
\end{array}
$$

We also introduce the notation $(\cdot, \cdot)_{h}$ for the $L^{2}\left(\Omega_{h}\right)$ inner product and the approximate sesquilinear form

$$
\left(\rho u_{h}, v_{h}\right)_{h}=\int_{\Omega_{h}} \rho u_{h} \bar{v}_{h}
$$

Remark 3.1. If $\Omega_{-}$is not convex we need an extension of $1 / \sigma$ and $\rho$ to $\Omega_{h} \backslash \Omega_{-}$. For well-posedness and convergence of our numerical method the only requirements are that $\|1 / \sigma\|_{\infty, \Omega_{h}} \leq C\|1 / \sigma\|_{\infty, \Omega_{-}}$and $\|\rho\|_{\infty, \Omega_{h}} \leq$ $C\|\rho\|_{\infty, \Omega_{-}}$and therefore they can be simply extended by zero at this stage. Later, in the convergence analysis we will take a bounded extension of $\sigma$ satisfying $\|1 / \sigma\|_{1, \infty, \Omega_{h}} \leq C\|1 / \sigma\|_{1, \infty, \Omega_{-}}$to guarantee the $\mathcal{O}(h+H)$ behaviour of the error in Theorem 8.2. Furthermore, to obtain a fully discrete method based on quadrature rules preserving the same convergence properties, we would also require the regularity condition $\rho \in W^{1, \infty}\left(\Omega_{-}\right)$ and consider an extension such that $\|\rho\|_{1, \infty, \Omega_{h}} \leq C\|\rho\|_{1, \infty, \Omega_{-}}$. Indeed, being more precise, what we need is that $\sigma$ and $\rho$ will be piecewise $W^{1, \infty}$-smooth, provided that the sequence of triangulations respects their discontinuity jumps.

Finally, we also need a false trace to approximate $\gamma$. We first consider the space $Z_{h} \subset H^{-1 / 2}$ of periodic piecewise constant functions with breakpoints on $\left\{z_{j}\right\}$. Recall that $\left\{z_{j}\right\}$ is the set of parametric values such that $\left\{\mathbf{x}\left(z_{j}\right)\right\}$ are the exterior vertices of $\mathcal{T}_{h}$. Notice that $S_{H}$ and $T_{H}$ are defined on staggered grids and that $Z_{h}$ is defined on a completely different grid. We define the operator $\beta_{h}: X_{h} \rightarrow Z_{h}$ as follows. If $L_{j}$ is the segment $\left[\mathbf{x}\left(z_{j}\right), \mathbf{x}\left(z_{j+1}\right)\right]$, which is a side of a triangle with vertices on $\Gamma$, and $\widetilde{L}_{j}$ is the curved segment on $\Gamma$ between $\mathbf{x}\left(z_{j}\right)$ and $\mathbf{x}\left(z_{j+1}\right)$, then

$$
\left.\beta_{h}\left(\mathbf{p}_{h}\right)\right|_{\left(z_{j}, z_{j+1}\right)}:=\left.\frac{\operatorname{length}\left(L_{j}\right)}{\operatorname{length}\left(\widetilde{L}_{j}\right)}\left(\mathbf{p}_{h} \cdot \nu\right)\right|_{L_{j}}=\frac{1}{\operatorname{length}\left(\widetilde{L}_{j}\right)} \int_{L_{j}}\left(\mathbf{p}_{h} \cdot \nu\right)(\mathbf{y}) \mathrm{d} \gamma_{\mathbf{y}}
$$

The discrete trace is defined by

$$
\gamma_{h}:=\left|\mathbf{x}^{\prime}\right| \beta_{h}
$$

The numerical method we propose is:

$$
\begin{array}{|ll}
\mathbf{p}_{h} \in X_{h}, \quad u_{h} \in L_{h}, \quad \xi_{H} \in S_{H}, \quad \psi_{H} \in T_{H}, & \\
a_{h}\left(\mathbf{p}_{h}, \mathbf{q}_{h}\right)+b_{h}^{*}\left(u_{h}, \mathbf{q}_{h}\right)-\left\langle\xi_{H}, \gamma_{h} \mathbf{q}_{h}\right\rangle=0, & \forall \mathbf{q}_{h} \in X_{h}, \\
b_{h}\left(\mathbf{p}_{h}, v_{h}\right)+\imath \omega\left(\rho u_{h}, v_{h}\right)_{h}=0, & \forall v_{h} \in L_{h}, \\
\left\langle\gamma_{h} \mathbf{p}_{h}, \eta_{H}\right\rangle+\sigma_{0}\left\langle\frac{1}{2} \psi_{H}-J_{\lambda} \psi_{H}, \eta_{H}\right\rangle=\left\langle g_{1}, \eta_{H}\right\rangle, & \forall \eta_{H} \in S_{H}, \\
-\left\langle\xi_{H}, \varphi_{H}\right\rangle+\left\langle V_{\lambda} \psi_{H}, \varphi_{H}\right\rangle=-\left\langle g_{0}, \varphi_{H}\right\rangle, & \forall \varphi_{H} \in T_{H} .
\end{array}
$$




\section{Abstract analysis of the Discretization}

The analysis of our numerical method, that is carried out in Sections $5-8$, follows two steps. We first show stability and convergence of the discretization when applied to the principal part of the operator. This is done by comparison with a related unperturbed Galerkin scheme. Then, by compactness arguments we will obtain stability and convergence of the method applied to the global operator.

In this section we deal with some properties and concepts on stability and convergence in the abstract setting in which our method falls. Our goal is to give conditions guaranteeing stability and some convergence properties of a perturbed Galerkin method by comparing it with a related (proper) Galerkin scheme. We use here some ideas that were introduced in a simpler setting in [40]. Similar results, for elliptic bilinear forms, were introduced in [24] to analyze the error of isoparametric finite elements. For many approximation results in this line, we refer to the classical monograph [2].

Let $V$ be a Hilbert space and $a: V \times V \rightarrow \mathbb{C}$ a sesquilinear form such that the operator $A: V \rightarrow V$ given by

$$
(A u, v)=a(u, v), \quad \forall u, v \in V
$$

is invertible. Given a conjugate linear form $\ell$, we consider the problem

$$
\mid \begin{aligned}
& u \in V, \\
& a(u, v)=\ell(v), \quad \forall v \in V .
\end{aligned}
$$

This problem is then well-posed. We set $M:=\|A\|$.

Let $V_{h}$ be a finite dimensional space (a sequence of them), endowed with an inner product norm $\|\cdot\|_{h}$ and let $a_{h}: V_{h} \times V_{h} \rightarrow \mathbb{C}$ and $\ell_{h}: V_{h} \rightarrow \mathbb{C}$ be respectively sesquilinear and conjugate linear forms, uniformly bounded in $V_{h}$, i.e. for all $h$

$$
\begin{array}{rlrl} 
& \left|a_{h}\left(u_{h}, v_{h}\right)\right| \leq M_{0}\left\|u_{h}\right\|_{h}\left\|v_{h}\right\|_{h}, & & \forall u_{h}, v_{h} \in V_{h}, \\
\left|\ell_{h}\left(u_{h}\right)\right| & \leq C_{0}\left\|u_{h}\right\|_{h}, & \forall u_{h} \in V_{h} .
\end{array}
$$

In principle, $V_{h} \not \subset V$, that is, $V_{h}$ could be totally unrelated to $V$. The discretization is

$$
\mid \begin{aligned}
& u_{h} \in V_{h}, \\
& a_{h}\left(u_{h}, v_{h}\right)=\ell_{h}\left(v_{h}\right), \quad \forall v_{h} \in V_{h} .
\end{aligned}
$$

We further assume the existence of a sequence of subspaces $\widetilde{V}_{h} \subset V$ such that $\operatorname{dim} \widetilde{V}_{h}=\operatorname{dim} V_{h}$ and that there exists an isomorphism $\Theta_{h}: V_{h} \rightarrow \widetilde{V}_{h}$ such that for all $h$

$$
C_{1}\left\|v_{h}\right\|_{h} \leq\left\|\Theta_{h} v_{h}\right\| \leq C_{2}\left\|v_{h}\right\|_{h}, \quad \forall v_{h} \in V_{h}
$$

We finally introduce the Galerkin scheme for problem (4.1)

$$
\mid \begin{aligned}
& \widetilde{u}_{h} \in \widetilde{V}_{h}, \\
& a\left(\widetilde{u}_{h}, \widetilde{v}_{h}\right)=\ell\left(\widetilde{v}_{h}\right), \quad \forall \widetilde{v}_{h} \in \widetilde{V}_{h} .
\end{aligned}
$$

Theorem 4.1. Assume that there exists a function $\varepsilon$ such that $\varepsilon(h) \rightarrow 0$ and

$$
\left|a_{h}\left(u_{h}, v_{h}\right)-a\left(\Theta_{h} u_{h}, \Theta_{h} v_{h}\right)\right| \leq \varepsilon(h)\left\|u_{h}\right\|_{h}\left\|v_{h}\right\|_{h}, \quad \forall u_{h}, v_{h} \in V_{h} .
$$


Then, the following assertions are equivalent:

(a) There exists $\alpha>0$ such that for $h$ small enough

$$
\sup _{0 \neq \widetilde{v}_{h} \in \widetilde{V}_{h}} \frac{\left|a\left(\widetilde{u}_{h}, \widetilde{v}_{h}\right)\right|}{\left\|\widetilde{v}_{h}\right\|} \geq \alpha\left\|\widetilde{u}_{h}\right\|, \quad \forall \widetilde{u}_{h} \in \widetilde{V}_{h} .
$$

(b) There exists $\beta>0$ such that for $h$ small enough

$$
\sup _{0 \neq v_{h} \in V_{h}} \frac{\left|a_{h}\left(u_{h}, v_{h}\right)\right|}{\left\|v_{h}\right\|_{h}} \geq \beta\left\|u_{h}\right\|_{h}, \quad \forall u_{h} \in V_{h} .
$$

Proof. Let $\widetilde{A}_{h}: \widetilde{V}_{h} \rightarrow \widetilde{V}_{h}$ and $A_{h}: V_{h} \rightarrow V_{h}$ be given by

$$
\begin{aligned}
\left(\widetilde{A}_{h} \widetilde{u}_{h}, \widetilde{v}_{h}\right) & =a\left(\widetilde{u}_{h}, \widetilde{v}_{h}\right), & \forall \widetilde{u}_{h}, \widetilde{v}_{h} \in \widetilde{V}_{h}, \\
\left(A_{h} u_{h}, v_{h}\right)_{h} & =a_{h}\left(u_{h}, v_{h}\right), & \forall u_{h}, v_{h} \in V_{h} .
\end{aligned}
$$

Let also $\Theta_{h}^{*}: \widetilde{V}_{h} \rightarrow V_{h}$ be the adjoint of $\Theta_{h}$ :

$$
\left(\Theta_{h}^{*} \widetilde{u}_{h}, v_{h}\right)_{h}=\left(\widetilde{u}_{h}, \Theta_{h} v_{h}\right), \quad \forall \widetilde{u}_{h} \in \widetilde{V}_{h}, v_{h} \in V_{h}
$$

Then $a\left(\Theta_{h} u_{h}, \Theta_{h} v_{h}\right)=\left(\Theta_{h}^{*} \widetilde{A}_{h} \Theta_{h} u_{h}, v_{h}\right)_{h}$ and (4.4) reads $\left\|A_{h}-\Theta_{h}^{*} \widetilde{A}_{h} \Theta_{h}\right\|_{h} \leq \varepsilon(h)$, where $\|\cdot\|_{h}$ is the operator norm in $\mathcal{L}\left(V_{h}\right)$.

Properties (a) and (b) are respectively equivalent to

$$
\begin{gathered}
\left\|\widetilde{A}_{h} \widetilde{u}_{h}\right\| \geq \alpha\left\|\widetilde{u}_{h}\right\|, \quad \forall \widetilde{u}_{h} \in \widetilde{V}_{h}, \\
\left\|A_{h} u_{h}\right\|_{h} \geq \beta\left\|u_{h}\right\|_{h}, \quad \forall u_{h} \in V_{h} .
\end{gathered}
$$

If (a) holds, then

$$
\left\|\Theta_{h}^{*} \widetilde{A}_{h} \Theta_{h} u_{h}\right\|_{h} \geq C_{1}\left\|\widetilde{A}_{h} \Theta_{h} u_{h}\right\| \geq \alpha C_{1}\left\|\Theta_{h} u_{h}\right\| \geq \alpha C_{1}^{2}\left\|u_{h}\right\|_{h}, \quad \forall u_{h} \in V_{h} .
$$

At the same time, if $\left\|\Theta_{h}^{*} \widetilde{A}_{h} \Theta_{h} u_{h}\right\|_{h} \geq \gamma\left\|u_{h}\right\|_{h}$, for all $u_{h} \in V_{h}$, then

$$
\left\|\widetilde{A}_{h} \widetilde{u}_{h}\right\| \geq\left(1 / C_{2}\right)\left\|\Theta_{h}^{*} \widetilde{A}_{h} \Theta_{h} \Theta_{h}^{-1} \widetilde{u}_{h}\right\|_{h} \geq\left(\gamma / C_{2}\right)\left\|\Theta_{h}^{-1} \widetilde{u}_{h}\right\|_{h} \geq\left(\gamma / C_{2}^{2}\right)\left\|\widetilde{u}_{h}\right\|, \quad \forall \widetilde{u}_{h} \in \widetilde{V}_{h}
$$

The proof is now straightforward. Condition (a) implies that

$$
\left\|A_{h} u_{h}\right\|_{h} \geq\left\|\Theta_{h}^{*} \widetilde{A}_{h} \Theta_{h} u_{h}\right\|_{h}-\varepsilon(h)\left\|u_{h}\right\|_{h} \geq(\gamma-\varepsilon(h))\left\|u_{h}\right\|_{h}, \quad \forall u_{h} \in V_{h},
$$

and therefore (b) holds. On the other hand, if (b) holds, then $\left\|\Theta_{h}^{*} \widetilde{A}_{h} \Theta_{h} u_{h}\right\|_{h} \geq(\beta-\varepsilon(h))\left\|u_{h}\right\|_{h}$, for all $u_{h} \in V_{h}$, and condition (a) holds.

Theorem 4.2. Assume that conditions (4.4) and (4.5) are satisfied. Then, there exists $C>0$ such that for $h$ small enough

where

$$
\left\|u-\Theta_{h} u_{h}\right\| \leq C\left[\inf _{\widetilde{v}_{h} \in \widetilde{V}_{h}}\left\|u-\widetilde{v}_{h}\right\|+\delta(h)+\varepsilon(h)\|u\|\right]
$$

$$
\delta(h):=\sup _{0 \neq w_{h} \in V_{h}} \frac{\left|\ell\left(\Theta_{h} w_{h}\right)-\ell_{h}\left(w_{h}\right)\right|}{\left\|w_{h}\right\|_{h}} .
$$


Proof. The result follows readily from a very simple argument in the spirit of the first Strang lemma. By Theorem 4.1, for arbitrary $\widetilde{v}_{h}=\Theta_{h} v_{h} \in \widetilde{V}_{h}\left(v_{h} \in V_{h}\right)$ we have

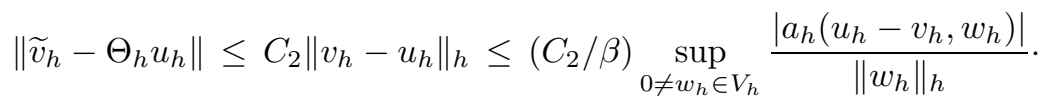

Let $\widetilde{u}_{h}$ be the solution to problem (4.3). Then, for all $w_{h} \in V_{h}$ it follows that

$$
a_{h}\left(u_{h}-v_{h}, w_{h}\right)=\ell_{h}\left(w_{h}\right)-\ell\left(\Theta_{h} w_{h}\right)+a\left(\widetilde{u}_{h}, \Theta_{h} w_{h}\right)-a_{h}\left(\Theta_{h}^{-1} \widetilde{u}_{h}, w_{h}\right)+a_{h}\left(\Theta_{h}^{-1} \widetilde{u}_{h}-v_{h}, w_{h}\right),
$$

and thus

$$
\begin{aligned}
\left|a_{h}\left(u_{h}-v_{h}, w_{h}\right)\right| & \leq \delta(h)\left\|w_{h}\right\|_{h}+\varepsilon(h)\left\|\Theta_{h}^{-1} \widetilde{u}_{h}\right\|_{h}\left\|w_{h}\right\|_{h}+M_{0}\left\|\Theta_{h}^{-1} \widetilde{u}_{h}-v_{h}\right\|_{h}\left\|w_{h}\right\|_{h} \\
& \leq\left[\delta(h)+\left(\varepsilon(h) / C_{1}\right)\left\|\widetilde{u}_{h}\right\|+\left(M_{0} / C_{1}\right)\left\|\widetilde{u}_{h}-\widetilde{v}_{h}\right\|\right]\left\|w_{h}\right\|_{h} .
\end{aligned}
$$

Finally,

$$
\begin{aligned}
\left\|u-\Theta_{h} u_{h}\right\| & \leq\left\|u-\widetilde{v}_{h}\right\|+\left\|\widetilde{v}_{h}-\Theta_{h} u_{h}\right\| \\
& \leq\left(1+C_{2} M_{0} M / C_{1} \alpha \beta\right)\left\|u-\widetilde{v}_{h}\right\|+\left(C_{2} / \alpha\right) \delta(h)+\left(C_{2} M / C_{1} \alpha \beta\right) \varepsilon(h)\|u\|,
\end{aligned}
$$

which finishes the proof.

\section{Discrete Well-POSEDNESS FOR THE PRINCIPAl PART}

The aim of this section is to prove that the discrete method applied to the operator $\mathcal{A}_{0}$ introduced in definition (2.5) (i.e. to the principal part of $\mathcal{A}$ ) satisfies a discrete inf-sup condition.

We write $|\cdot|_{h}$ and $\|\cdot\|_{\operatorname{div}, h}$ for the usual norms in the spaces $L^{2}\left(\Omega_{h}\right)$ and $H\left(\operatorname{div}, \Omega_{h}\right)$ respectively. Then, it follows that (recall the comments on $\sigma$ and $\rho$ in Sect. 3)

$$
\begin{aligned}
\left|a_{h}\left(\mathbf{p}_{h}, \mathbf{q}_{h}\right)\right| & \leq C\left\|\mathbf{p}_{h}\right\|_{\operatorname{div}, h}\left\|\mathbf{q}_{h}\right\|_{\operatorname{div}, h}, \\
\left|b_{h}\left(\mathbf{p}_{h}, v_{h}\right)\right| & \leq\left\|\mathbf{p}_{h}\right\|_{\operatorname{div}, h}\left\|v_{h}\right\|_{h} \\
\left|\left(\rho u_{h}, v_{h}\right)_{h}\right| & \leq C\left|u_{h}\right|_{h}\left|v_{h}\right|_{h} .
\end{aligned}
$$

We introduce the discrete operators $A_{h}: X_{h} \rightarrow X_{h}$ and $\rho_{h}: L_{h} \rightarrow L_{h}$ given by

$$
\begin{aligned}
\left(A_{h} \mathbf{p}_{h}, \mathbf{q}_{h}\right)_{\operatorname{div}, h} & =a_{h}\left(\mathbf{p}_{h}, \mathbf{q}_{h}\right), \quad \forall \mathbf{p}_{h}, \mathbf{q}_{h} \in X_{h}, \\
\left(\rho_{h} u_{h}, v_{h}\right)_{h} & =\left(\rho u_{h}, v_{h}\right)_{h}, \quad \forall u_{h}, v_{h} \in L_{h} .
\end{aligned}
$$

Here $(\cdot, \cdot)_{h}$ and $(\cdot, \cdot)_{\operatorname{div}, h}$ are the inner products in $L^{2}\left(\Omega_{h}\right)$ and $H\left(\operatorname{div}, \Omega_{h}\right)$. Note that the operator $\rho_{h}$ is simply multiplication by $\rho$ and orthogonal projection. Similarly, we define $B_{h}: X_{h} \rightarrow L_{h}$ by the relation

$$
\left(B_{h} \mathbf{p}_{h}, v_{h}\right)_{h}=b_{h}\left(\mathbf{p}_{h}, v_{h}\right), \quad \forall \mathbf{p}_{h} \in X_{h}, v_{h} \in L_{h}
$$


Since $\nabla \cdot \mathbf{p}_{h} \in L_{h}$ for all $\mathbf{p}_{h} \in X_{h}$ it is straightforward to show that $B_{h} \mathbf{p}_{h}=\nabla \cdot \mathbf{p}_{h}$. We also define $R_{h, H}$ : $X_{h} \rightarrow S_{H}^{*}, M_{H}: S_{H} \rightarrow T_{H}^{*}$ and $V_{H}: T_{H} \rightarrow T_{H}^{*}$ by

$$
\begin{aligned}
\left\langle R_{h, H} \mathbf{p}_{h}, \eta_{H}\right\rangle & =\left\langle\gamma_{h} \mathbf{p}_{h}, \eta_{H}\right\rangle, & & \forall \mathbf{p}_{h} \in X_{h}, \eta_{H} \in S_{H}, \\
\left\langle M_{H} \xi_{H}, \varphi_{H}\right\rangle & =\left\langle\xi_{H}, \varphi_{H}\right\rangle, & & \forall \xi_{H} \in S_{H}, \varphi_{H} \in T_{H}, \\
\left\langle V_{H} \psi_{H}, \varphi_{H}\right\rangle & =\left\langle V_{\lambda} \psi_{H}, \varphi_{H}\right\rangle, & & \forall \psi_{H}, \varphi_{H} \in T_{H},
\end{aligned}
$$

where $S_{H}^{*}$ and $T_{H}^{*}$ are the antidual spaces of $S_{H}$ and $T_{H}$. Finally, we define the adjoint operators $B_{h}^{*}: L_{h} \rightarrow X_{h}$ and $R_{h, H}^{*}: S_{H} \rightarrow X_{h}$ by

$$
\begin{aligned}
\left(\mathbf{p}_{h}, B_{h}^{*} v_{h}\right)_{\operatorname{div}, h}=\left(B_{h} \mathbf{p}_{h}, v_{h}\right)_{h}, & \forall \mathbf{p}_{h} \in X_{h}, \quad v_{h} \in L_{h}, \\
\left(\mathbf{p}_{h}, R_{h, H}^{*} \eta_{H}\right)_{\operatorname{div}, h}=\left\langle\gamma_{h} \mathbf{p}_{h}, \eta_{H}\right\rangle, & \forall \mathbf{p}_{h} \in X_{h}, \eta_{H} \in S_{H} .
\end{aligned}
$$

Proposition 5.1. The operators $A_{h}, B_{h}, \rho_{h}, \rho_{h}^{-1}, V_{H}, V_{H}^{-1}, M_{H}$ and $M_{H}^{-1}$ are uniformly bounded with respect to the corresponding discrete norms.

Proof. The result for $A_{h}, B_{h}, \rho_{h}, \rho_{h}^{-1}, V_{H}$ and $M_{H}$ is elementary. Since $V_{\lambda}$ is elliptic (see inequality (2.1)), then $V_{H}^{-1}$ is also uniformly bounded. Uniform boundedness of $M_{H}^{-1}$ is equivalent to

$$
\sup _{0 \neq \varphi_{H} \in T_{H}} \frac{\left|\left\langle\xi_{H}, \varphi_{H}\right\rangle\right|}{\left\|\varphi_{H}\right\|_{-1 / 2}} \geq C\left\|\xi_{H}\right\|_{1 / 2}, \quad \forall \xi_{H} \in S_{H}
$$

In its turn, this is a straightforward consequence of [39], Proposition 6.4.

Proposition 5.2. There exists $C>0$, independent of $h$, such that

$$
\left\|\gamma_{h} \mathbf{q}_{h}\right\|_{-1 / 2} \leq C\left\|\mathbf{q}_{h}\right\|_{\operatorname{div}, h}, \quad \forall \mathbf{q}_{h} \in X_{h} .
$$

Therefore, $R_{h, H}$ is uniformly bounded.

Proof. This result follows from Proposition 7.1, which requires the introduction of curved finite elements for its proof. These elements will be introduced in Section 6 .

Proposition 5.3. The operator

$$
\mathcal{A}_{0}^{h, H}:=\left[\begin{array}{cccc}
A_{h} & B_{h}^{*} & -R_{h, H}^{*} & 0 \\
B_{h} & \imath \omega \rho_{h} & 0 & 0 \\
R_{h, H} & 0 & 0 & \frac{\sigma_{0}}{2} M_{H}^{*} \\
0 & 0 & -M_{H} & V_{H}
\end{array}\right]: X_{h} \times L_{h} \times S_{H} \times T_{H} \rightarrow X_{h} \times L_{h} \times S_{H}^{*} \times T_{H}^{*}
$$

has a uniformly bounded inverse with respect to the product discrete norm. 
Proof. We proceed as in the continuous case. We first decompose $\mathcal{A}_{0}^{h, H}$ as

$$
\left[\begin{array}{cccc}
I & -\frac{\imath}{\omega} B_{h}^{*} \rho_{h}^{-1} & -\frac{2}{\sigma_{0}} R_{h, H}^{*} M_{H}^{-1} V_{H}\left(M_{H}^{-1}\right)^{*} & 0 \\
0 & I & 0 & 0 \\
0 & 0 & I & \frac{\sigma_{0}}{2} M_{H}^{*} V_{H}^{-1} \\
0 & 0 & 0 & I
\end{array}\right]\left[\begin{array}{cccc}
Q_{h, H} & 0 & 0 & 0 \\
B_{h} & \imath \omega \rho_{h} & 0 & 0 \\
R_{h, H} & 0 & \frac{\sigma_{0}}{2} M_{H}^{*} V_{H}^{-1} M_{H} & 0 \\
0 & 0 & -M_{H} & V_{H}
\end{array}\right]
$$

where

$$
Q_{h, H}:=A_{h}+(\imath / \omega) B_{h}^{*} \rho_{h}^{-1} B_{h}+\left(2 / \sigma_{0}\right) R_{h, H}^{*} M_{H}^{-1} V_{H}\left(M_{H}^{-1}\right)^{*} R_{h, H}
$$

By Propositions 5.1 and 5.2, the inverse of the left-most operator in the decomposition above is uniformly bounded. All the off-diagonal operators in the right-most matrix are also uniformly bounded as well as $\rho_{h}^{-1}$, $V_{H}^{-1}$ and $\left(M_{H}^{*} V_{H}^{-1} M_{H}\right)^{-1}=M_{H}^{-1} V_{H}\left(M_{H}^{-1}\right)^{*}$. Hence, we only have to show that $Q_{h, H}$ has a uniformly bounded inverse. To do this, we will prove as in the continuous case that

$$
\operatorname{Re}\left[(1-\imath)\left(Q_{h, H} \mathbf{p}_{h}, \mathbf{p}_{h}\right)_{\operatorname{div}, h}\right] \geq \alpha\left\|\mathbf{p}_{h}\right\|_{\text {div }, h}^{2}, \quad \forall \mathbf{p}_{h} \in X_{h} .
$$

Since $B_{h} \mathbf{p}_{h}=\nabla \cdot \mathbf{p}_{h}$,

$$
\operatorname{Re}\left[(1-\imath)\left(\left(A_{h}+(\imath / \omega) B_{h}^{*} \rho_{h}^{-1} B_{h}\right) \mathbf{p}_{h}, \mathbf{p}_{h}\right)_{\operatorname{div}, h}\right]=\int_{\Omega_{h}}(1 / \sigma)\left|\mathbf{p}_{h}\right|^{2}+\int_{\Omega_{h}}(1 / \omega \rho)\left|\nabla \cdot \mathbf{p}_{h}\right|^{2},
$$

and from inequality (2.1) we deduce that

$$
\begin{aligned}
\operatorname{Re}\left[( 1 - \imath ) \left(R_{h, H}^{*} M_{H}^{-1} V_{H}\right.\right. & \left.\left.\left(M_{H}^{-1}\right)^{*} R_{h, H} \mathbf{p}_{h}, \mathbf{p}_{h}\right)_{\operatorname{div}, h}\right] \\
& =\operatorname{Re}\left[(1-\imath)\left\langle V_{\lambda}\left(M_{H}^{-1}\right)^{*} R_{h, H} \mathbf{p}_{h},\left(M_{H}^{-1}\right)^{*} R_{h, H} \mathbf{p}_{h}\right\rangle\right] \geq 0 .
\end{aligned}
$$

The result follows then from the hypotheses on $\sigma$ and $\rho$.

\section{A conforming Curved Galerkin method}

Here we propose a proper Galerkin discretization to solve numerically problem (2.3). Our aim is not to use it with practical purposes but to take it as a link between problems (2.3) and (3.1), as in the abstract setting of Section 4. However, as hinted in the introduction, we could use these ideas as a starting point for the construction of higher order methods with curved triangles as in [30,31,35]. We begin by recalling the curved Raviart-Thomas elements of order zero and proving some properties that will be used later in the analysis of the method (3.1). For an exhaustive description and analysis of curved elements we refer to [44-46].

\subsection{Curved Raviart-Thomas elements}

We first gather here some definitions and notations related to the classical $\mathcal{R} \mathcal{T}_{0}$ space, defined over (straight) triangles. For any triangle $T$ we consider the space

$$
\mathbb{P}(T):=\left\{\mathbf{p}: T \rightarrow \mathbb{C}^{2} \mid \mathbf{p}(\mathbf{x})=\mathbf{a}+\alpha \mathbf{x}, \quad \mathbf{a} \in \mathbb{C}^{2}, \alpha \in \mathbb{C}\right\} .
$$

Given any side $L$ of $T$, we denote

$$
\Phi_{L}(\mathbf{p}):=\int_{L} \mathbf{p} \cdot \nu
$$


For $T$ given, we consider an invertible affine map from the reference triangle $\widehat{T}$ into $T, F_{T}: \widehat{T} \rightarrow T$. Let $B_{T}:=\mathrm{D} F_{T}$ be its Jacobian matrix and $J_{T}:=\operatorname{det} B_{T}$. It is well known (see for instance [4], Chap. 3) that the Piola transform $\mathcal{P}_{T}: \mathbb{P}(\widehat{T}) \rightarrow \mathbb{P}(T)$ given by

$$
\mathcal{P}_{T} \widehat{\mathbf{p}}:=\frac{1}{J_{T}} B_{T} \widehat{\mathbf{p}} \circ F_{T}^{-1}
$$

is well-defined, invertible and satisfies

$$
\Phi_{L}\left(\mathcal{P}_{T} \widehat{\mathbf{p}}\right)=\Phi_{\widehat{L}}(\widehat{\mathbf{p}}), \quad \forall \widehat{\mathbf{p}} \in \mathbb{P}(\widehat{T}),
$$

where $L=F_{T}(\widehat{L})$. Moreover,

$$
\nabla \cdot \mathcal{P}_{T} \widehat{\mathbf{p}}=\frac{1}{J_{T}} \nabla \cdot \widehat{\mathbf{p}}, \quad \forall \widehat{\mathbf{p}} \in \mathbb{P}(\widehat{T}) .
$$

Let $\widetilde{T}$ be a curved triangle with a single side on $\Gamma$ and denote $T$ to the underlying straight triangle. We consider an invertible map $\widetilde{F}_{T}: \widehat{T} \rightarrow \widetilde{T}, \widetilde{B}_{T}:=\mathrm{D} \widetilde{F}_{T}$ and $\widetilde{J}_{T}:=\operatorname{det} \widetilde{B}_{T}$. Conditions guaranteeing the existence of $\widetilde{F}_{T}$ and related properties can be found in $[31,44-46]$. We then define

$$
\widetilde{\mathcal{P}}_{T} \widehat{\mathbf{p}}:=\left(\frac{1}{\widetilde{J}_{T}} \widetilde{B}_{T} \widehat{\mathbf{p}}\right) \circ \widetilde{F}_{T}^{-1},
$$

and introduce the space $\mathbb{P}(\widetilde{T}):=\left\{\widetilde{\mathcal{P}}_{T} \widehat{\mathbf{p}} \mid \widehat{\mathbf{p}} \in \mathbb{P}(\widehat{T})\right\}$. By ([4], Chap. 3, (1.47)), we have the following relation, similar to equality $(6.1)$,

$$
\nabla \cdot \widetilde{\mathcal{P}}_{T} \widehat{\mathbf{p}}=\frac{1}{\widetilde{J}_{T}} \nabla \cdot \widehat{\mathbf{p}}, \quad \forall \widehat{\mathbf{p}} \in \mathbb{P}(\widehat{T}) .
$$

We group in the next result some bounds extracted from ([44], Chap. 22), (see also [31] and references therein). For brevity, we write

$$
\Theta_{T}:=\widetilde{B}_{T}-B_{T}=\mathrm{D} \widetilde{F}_{T}-\mathrm{D} F_{T}
$$

Proposition 6.1. The following bounds hold

$$
\begin{aligned}
&\left\|J_{T}\right\|_{\infty, \widehat{T}}+\left\|\widetilde{J}_{T}\right\|_{\infty, \widehat{T}}+\left\|\Theta_{T}\right\|_{\infty, \widehat{T}} \leq C h_{T}^{2},\left\|J_{T}^{-1}\right\|_{\infty, \widehat{T}}+\left\|\widetilde{J}_{T}^{-1}\right\|_{\infty, \widehat{T}} \leq C h_{T}^{-2}, \\
&\left\|B_{T}\right\|_{\infty, \widehat{T}}+\left\|\widetilde{B}_{T}\right\|_{\infty, \widehat{T}} \leq C h_{T}, \quad\left\|B_{T}^{-1}\right\|_{\infty, \widehat{T}}+\left\|\widetilde{B}_{T}^{-1}\right\|_{\infty, \widehat{T}} \leq C h_{T}^{-1} .
\end{aligned}
$$

For derivatives we have

$$
\left|\widetilde{J}_{T}\right|_{1, \infty, \widehat{T}} \leq C h_{T}^{3}, \quad\left|\widetilde{J}_{T}^{-1}\right|_{1, \infty, \widehat{T}} \leq C h_{T}^{-1}, \quad\left|\widetilde{B}_{T}\right|_{1, \infty, \widehat{T}} \leq C h_{T}^{2}, \quad\left|\widetilde{B}_{T}^{-1}\right|_{1, \infty, \widehat{T}} \leq C .
$$

Using ([4], Chap. 3, Lemma 1.6), and Proposition 6.1, the next result concerning the Piola transforms can be easily proven.

Proposition 6.2. There exist $C_{1}, C_{2}>0$, independent of $T$, such that

$$
\begin{aligned}
& C_{1}\|\widehat{\mathbf{p}}\|_{0, \widehat{T}} \leq\left\|\mathcal{P}_{T} \widehat{\mathbf{p}}\right\|_{0, T} \leq C_{2}\|\widehat{\mathbf{p}}\|_{0, \widehat{T}}, \quad \forall \widehat{\mathbf{p}} \in \mathbb{P}(\widehat{T}) \\
& C_{1}\|\widehat{\mathbf{p}}\|_{0, \widehat{T}} \leq\left\|\widetilde{\mathcal{P}_{T}} \widehat{\mathbf{p}}\right\|_{0, \widetilde{T}} \leq C_{2}\|\widehat{\mathbf{p}}\|_{0, \widehat{T}}, \quad \forall \widehat{\mathbf{p}} \in \mathbb{P}(\widehat{T})
\end{aligned}
$$

For the inverse transforms we have

$$
\begin{array}{ll}
\left\|\mathcal{P}_{T}^{-1} \mathbf{p}\right\|_{\text {div }, \widehat{T}} \leq C\|\mathbf{p}\|_{\text {div }, T}, & \forall \mathbf{p} \in \mathbb{P}(T), \\
\left\|\widetilde{\mathcal{P}}_{T}^{-1} \widetilde{\mathbf{p}}\right\|_{\text {div }, \widehat{T}} \leq C\|\widetilde{\mathbf{p}}\|_{\text {div }, \widetilde{T}}, \quad \forall \widetilde{\mathbf{p}} \in \mathbb{P}(\widetilde{T}) .
\end{array}
$$


Proposition 6.3. The transformation $\widetilde{\mathcal{P}}_{T} \mathcal{P}_{T}^{-1}: \mathbb{P}(T) \rightarrow \mathbb{P}(\widetilde{T})$ is uniformly bounded and has uniformly bounded inverse, that is,

$$
C_{1}\|\mathbf{p}\|_{\text {div }, T} \leq\left\|\widetilde{\mathcal{P}}_{T} \mathcal{P}_{T}^{-1} \mathbf{p}\right\|_{\text {div }, \widetilde{T}} \leq C_{2}\|\mathbf{p}\|_{\text {div }, T}, \quad \forall \mathbf{p} \in \mathbb{P}(T) .
$$

Furthermore,

$$
\Phi_{\widetilde{L}}\left(\widetilde{\mathcal{P}}_{T} \widehat{\mathbf{p}}\right)=\Phi_{\widehat{L}}(\widehat{\mathbf{p}}), \quad \forall \widehat{\mathbf{p}} \in \mathbb{P}(\widehat{T}) .
$$

Finally, if $\widetilde{\mathbf{p}} \in \mathbb{P}(\widetilde{T})$, then $\left.\widetilde{\mathbf{p}} \cdot \nu\right|_{\widetilde{L}} \in \mathbf{P}_{0}$.

Proof. By Proposition 6.2,

$$
\left\|\widetilde{\mathcal{P}}_{T} \mathcal{P}_{T}^{-1} \mathbf{p}\right\|_{0, \widetilde{T}} \leq C\left\|\mathcal{P}_{T}^{-1} \mathbf{p}\right\|_{0, \widehat{T}} \leq C^{\prime}\|\mathbf{p}\|_{0, T}, \quad \forall \mathbf{p} \in \mathbb{P}(T) .
$$

From relations (6.1) and (6.2) and Proposition 6.1, we also deduce that

$$
\left\|\nabla \cdot\left(\widetilde{\mathcal{P}}_{T} \mathcal{P}_{T}^{-1} \mathbf{p}\right)\right\|_{0, \widetilde{T}}=\left\|\left(J_{T} / \widetilde{J}_{T}\right) \nabla \cdot \mathbf{p}\right\|_{0, T} \leq C\|\nabla \cdot \mathbf{p}\|_{0, T}, \quad \forall \mathbf{p} \in \mathbb{P}(T) .
$$

Thus estimates (6.8) and (6.9) prove the second inequality in (6.7). Uniform boundedness of the inverse transform, $\left(\widetilde{\mathcal{P}}_{T} \mathcal{P}_{T}^{-1}\right)^{-1}=\mathcal{P}_{T} \widetilde{\mathcal{P}}_{T}^{-1}$, follows from similar arguments. The last two properties follow readily from [4], Lemma 1.5.

\subsection{Conforming curved elements}

Given a triangulation $\mathcal{T}_{h}$ of $\Omega_{-}$, we consider the curved triangulation $\widetilde{\mathcal{T}}_{h}$ with the same vertices as those of $\mathcal{T}_{h}$. We then define the space of piecewise constant functions on $\widetilde{\mathcal{T}}_{h}$,

$$
\widetilde{L}_{h}:=\left\{\widetilde{u}_{h} \in L^{2}\left(\Omega_{-}\right)\left|\widetilde{u}_{h}\right|_{\widetilde{T}} \in \mathbb{P}_{0}, \quad \forall \widetilde{T} \in \widetilde{\mathcal{T}}_{h}\right\},
$$

and the $\mathcal{R} \mathcal{T}_{0}$ space associated to the same curved triangulation,

$$
\widetilde{X}_{h}:=\left\{\widetilde{\mathbf{p}}_{h} \in H\left(\operatorname{div}, \Omega_{-}\right)\left|\widetilde{\mathbf{p}}_{h}\right|_{\widetilde{T}} \in \mathbb{P}(\widetilde{T}), \quad \forall \widetilde{T} \in \widetilde{\mathcal{T}}_{h}\right\} .
$$

The spaces on the boundary are not modified and we just take $\widetilde{S}_{H}:=S_{H}$ and $\widetilde{T}_{H}:=T_{H}$. With these new discrete spaces we consider the Galerkin scheme

$$
\mid \begin{array}{ll}
\widetilde{\mathbf{p}}_{h} \in \widetilde{X}_{h}, \quad \widetilde{u}_{h} \in \widetilde{L}_{h}, \quad \widetilde{\xi}_{H} \in \widetilde{S}_{H}, \quad \widetilde{\psi}_{H} \in \widetilde{T}_{H}, & \\
a\left(\widetilde{\mathbf{p}}_{h}, \widetilde{\mathbf{q}}_{h}\right)+b^{*}\left(\widetilde{u}_{h}, \widetilde{\mathbf{q}}_{h}\right)-\left\langle\widetilde{\xi}_{H}, \gamma \widetilde{\mathbf{q}}_{h}\right\rangle=0, & \forall \widetilde{\mathbf{q}}_{h} \in \widetilde{X}_{h}, \\
b\left(\widetilde{\mathbf{p}}_{h}, \widetilde{v}_{h}\right)+\imath \omega\left(\rho \widetilde{u}_{h}, \widetilde{v}_{h}\right)=0, & \widetilde{L}_{h}, \\
\left\langle\gamma \widetilde{\mathbf{p}}_{h}, \widetilde{\eta}_{H}\right\rangle+\sigma_{0}\left\langle\frac{1}{2} \widetilde{\psi}_{H}-J_{\lambda} \widetilde{\psi}_{H}, \widetilde{\eta}_{H}\right\rangle=\left\langle g_{1}, \widetilde{\eta}_{H}\right\rangle, & \forall \widetilde{\eta}_{H} \in \widetilde{S}_{H}, \\
-\left\langle\widetilde{\xi}_{H}, \widetilde{\varphi}_{H}\right\rangle+\left\langle V_{\lambda} \widetilde{\psi}_{H}, \widetilde{\varphi}_{H}\right\rangle=-\left\langle g_{0}, \widetilde{\varphi}_{H}\right\rangle, & \forall \widetilde{\varphi}_{H} \in \widetilde{T}_{H},
\end{array}
$$

to approximate problem (2.3). To fall into the abstract setting of Section 4, we define

$$
\Theta_{h, H}: X_{h} \times L_{h} \times S_{H} \times T_{H} \rightarrow \widetilde{X}_{h} \times \widetilde{L}_{h} \times \widetilde{S}_{H} \times \widetilde{T}_{H}
$$

by making nodal basis functions coincide, i.e. if

$$
\left(\widetilde{\mathbf{p}}_{h}, \widetilde{u}_{h}, \widetilde{\xi}_{H}, \widetilde{\psi}_{H}\right)=\Theta_{h, H}\left(\mathbf{p}_{h}, u_{h}, \xi_{H}, \psi_{H}\right),
$$


then for all $T \in \mathcal{T}_{h}$

$$
\left.\widetilde{u}_{h}\right|_{\widetilde{T}}=\left.u_{h}\right|_{T},\left.\quad \widetilde{\mathbf{p}}_{h}\right|_{\widetilde{T}}=\widetilde{\mathcal{P}}_{T} \mathcal{P}_{T}^{-1}\left(\left.\mathbf{p}_{h}\right|_{T}\right)
$$

and trivially $\widetilde{\xi}_{H}=\xi_{H}$ and $\widetilde{\psi}_{H}=\psi_{H}$.

Proposition 6.4. The operator $\Theta_{h, H}: X_{h} \times L_{h} \times S_{H} \times T_{H} \rightarrow \widetilde{X}_{h} \times \widetilde{L}_{h} \times \widetilde{S}_{H} \times \widetilde{T}_{H}$ is uniformly bounded with uniformly bounded inverse (the corresponding norms are the usual ones in the product spaces $H\left(\operatorname{div}, \Omega_{h}\right) \times$ $L^{2}\left(\Omega_{h}\right) \times H^{1 / 2} \times H^{-1 / 2}$ and $\left.H\left(\operatorname{div}, \Omega_{-}\right) \times L^{2}\left(\Omega_{-}\right) \times H^{1 / 2} \times H^{-1 / 2}\right)$.

Proof. The $H(\mathrm{div})$ part is a consequence of Proposition 6.3. The $L^{2}$ part follows from the fact that

$$
C_{1} \text { area }(T) \leq \operatorname{area}(\widetilde{T}) \leq C_{2} \text { area }(T),
$$

with $C_{1}$ and $C_{2}$ independent of $T$.

\section{COMPARISON OF STRAight AND CURVED ELEMENTS}

Our next purpose is to deal with the discrete sesquilinear forms appearing in the numerical method. We will compare them with the corresponding exact sesquilinear forms restricted to the spaces of curved elements introduced in Section 6. The type of analysis we will carry out here is developed in the spirit of the bounds in $[24,40]$ for isoparametric elements.

Proposition 7.1. Let $\mathbf{p}_{h} \in X_{h}$ and $\widetilde{\mathbf{p}}_{h} \in \widetilde{X}_{h}$ be the corresponding transformed curved discrete function. Then, $\gamma_{h} \mathbf{p}_{h}=\gamma \widetilde{\mathbf{p}}_{h}$ and therefore $\gamma_{h}$ is uniformly bounded.

Proof. Recalling the notations at the end of Section 3 and applying Proposition 6.3, we have that

$$
\left.\beta_{h} \mathbf{p}_{h}\right|_{\left(z_{j}, z_{j+1}\right)}=\left.\left(\widetilde{\mathbf{p}}_{h} \cdot \nu\right)\right|_{\widetilde{L}_{j}}=\left.\left(\widetilde{\mathbf{p}}_{h} \cdot \nu\right) \circ \mathbf{x}\right|_{\left(z_{j}, z_{j+1}\right)} \cdot
$$

The result follows readily from this equality.

Lemma 7.2. Let $V_{T}:=\left(\widetilde{J}_{T} / J_{T}\right) \widetilde{B}_{T}^{-1} B_{T}$. Then, there exists $C>0$, independent of $T$, such that

$$
\left\|I-V_{T}\right\|_{\infty, \widehat{T}} \leq C h_{T}
$$

Proof. Since $B_{T}=\widetilde{B}_{T}-\Theta_{T}$, then

$$
I-V_{T}=\left(1-\operatorname{det}\left(I+B_{T}^{-1} \Theta_{T}\right)\right) I+\operatorname{det}\left(I+B_{T}^{-1} \Theta_{T}\right) \widetilde{B}_{T}^{-1} \Theta_{T}
$$

and we apply Proposition 6.1 to prove the result.

Lemma 7.3. There exists $C>0$, independent of $T$, such that

$$
\left\|\widehat{\mathbf{p}} \circ F_{T}^{-1} \circ \widetilde{F}_{T}-\widehat{\mathbf{p}}\right\|_{1, \widehat{T}} \leq C h_{T}\|\nabla \cdot \widehat{\mathbf{p}}\|_{0, \widehat{T}}, \quad \forall \widehat{\mathbf{p}} \in \mathbb{P}(\widehat{T}) .
$$

Proof. If $\widehat{\mathbf{p}}(\widehat{\mathbf{x}})=\mathbf{a}+\alpha \widehat{\mathbf{x}} \in \mathbb{P}(\widehat{T})$, then $\alpha=\frac{1}{2} \nabla \cdot \widehat{\mathbf{p}}$ and therefore $|\alpha|=(1 / \sqrt{2})\|\nabla \cdot \widehat{\mathbf{p}}\|_{0, \widehat{T}}$. Since

$$
F_{T}^{-1} \circ \widetilde{F}_{T}(\widehat{\mathbf{x}})=\widehat{\mathbf{x}}+B_{T}^{-1} G_{T}(\widehat{\mathbf{x}}), \quad \forall \widehat{\mathbf{x}} \in \widehat{T}
$$

with $G_{T}:=\widetilde{F}_{T}-F_{T}$, it follows that

$$
\widehat{\mathbf{p}} \circ F_{T}^{-1} \circ \widetilde{F}_{T}-\widehat{\mathbf{p}}=\alpha B_{T}^{-1} G_{T}
$$


Notice that by definition of $G_{T},\left\|G_{T}\right\|_{\infty, \widehat{T}} \leq C h_{T}^{2}$ and by Proposition $6.1,\left|G_{T}\right|_{1, \infty, \widehat{T}} \leq C h_{T}^{2}$. Therefore,

$$
\left\|\widehat{\mathbf{p}} \circ F_{T}^{-1} \circ \widetilde{F}_{T}-\widehat{\mathbf{p}}\right\|_{1, \widehat{T}} \leq C|\alpha|\left\|B_{T}^{-1}\right\|_{\infty, \widehat{T}}\left\|G_{T}\right\|_{1, \infty, \widehat{T}} \leq C^{\prime} h_{T}\|\nabla \cdot \widehat{\mathbf{p}}\|_{0, \widehat{T}}
$$

Proposition 7.4. There exists $C>0$, independent of $h$, such that

$$
\left\|\widetilde{\mathbf{p}}_{h}-\mathbf{p}_{h}\right\|_{\mathrm{div}, \Omega_{-}} \leq C h\left\|\mathbf{p}_{h}\right\|_{\mathrm{div}, h}, \quad \forall \mathbf{p}_{h} \in X_{h},
$$

where $\widetilde{\mathbf{p}}_{h}$ is the curved discrete function transformed from $\mathbf{p}_{h}$ and in $\widetilde{T} \backslash T$ we understand $\mathbf{p}_{h}$ to have the same expression as in $T$.

Proof. For any triangle $T$, let $\widehat{\mathbf{p}}_{T}$ be such that

$$
\left.\widetilde{\mathbf{p}}_{h}\right|_{\widetilde{T}}=\widetilde{\mathcal{P}}_{T} \widehat{\mathbf{p}}_{T},\left.\quad \mathbf{p}_{h}\right|_{T}=\mathcal{P}_{T} \widehat{\mathbf{p}}_{T}
$$

Notice first that

$$
\widetilde{\mathcal{P}}_{T}^{-1} \mathcal{P}_{T} \widehat{\mathbf{p}}_{T}=\widetilde{\mathcal{P}}_{T}^{-1}\left(\frac{1}{J_{T}} B_{T} \widehat{\mathbf{p}}_{T} \circ F_{T}^{-1}\right)=V_{T}\left(\widehat{\mathbf{p}}_{T} \circ F_{T}^{-1} \circ \widetilde{F}_{T}\right),
$$

with $V_{T}$ as in Lemma 7.2. Then, by bound (6.4) and Lemmas 7.2 and 7.3 we obtain that

$$
\begin{aligned}
\left\|\mathbf{p}_{h}-\widetilde{\mathbf{p}}_{h}\right\|_{0, \widetilde{T}} & =\left\|\mathcal{P}_{T} \widehat{\mathbf{p}}_{T}-\widetilde{\mathcal{P}}_{T} \widehat{\mathbf{p}}_{T}\right\|_{0, \widetilde{T}} \leq C\left\|\widetilde{\mathcal{P}}_{T}^{-1} \mathcal{P}_{T} \widehat{\mathbf{p}}_{T}-\widehat{\mathbf{p}}_{T}\right\|_{0, \widehat{T}} \\
& \leq C\left(\left\|\left(I-V_{T}\right) \widehat{\mathbf{p}}_{T}\right\|_{0, \widehat{T}}+\left\|V_{T}\left(\widehat{\mathbf{p}}_{T} \circ F_{T}^{-1} \circ \widetilde{F}_{T}-\widehat{\mathbf{p}}_{T}\right)\right\|_{0, \widehat{T}}\right) \\
& \leq C\left(\left\|I-V_{T}\right\|_{\infty, \widehat{T}}\left\|\widehat{\mathbf{p}}_{T}\right\|_{0, \widehat{T}}+\left\|V_{T}\right\|_{\infty, \widehat{T}}\left\|\widehat{\mathbf{p}}_{T} \circ F_{T}^{-1} \circ \widetilde{F}_{T}-\widehat{\mathbf{p}}_{T}\right\|_{0, \widehat{T}}\right) \\
& \leq C_{1} h_{T}\left\|\widehat{\mathbf{p}}_{T}\right\|_{0, \widehat{T}}+C_{2} h_{T}\left\|\nabla \cdot \widehat{\mathbf{p}}_{T}\right\|_{0, \widehat{T}} \leq C_{3} h_{T}\left\|\widehat{\mathbf{p}}_{T}\right\|_{\mathrm{div}, \widehat{T}} .
\end{aligned}
$$

From inequality (6.5), it follows that

$$
\left\|\mathbf{p}_{h}-\widetilde{\mathbf{p}}_{h}\right\|_{0, \widetilde{T}} \leq C h_{T}\left\|\mathbf{p}_{h}\right\|_{\text {div }, T}
$$

On the other hand, by the relations (6.1) and (6.2),

$$
\left\|\nabla \cdot\left(\mathbf{p}_{h}-\widetilde{\mathbf{p}}_{h}\right)\right\|_{0, \widetilde{T}}=\left\|\left(1-J_{T} / \widetilde{J}_{T}\right) \nabla \cdot \mathbf{p}_{h}\right\|_{0, T} \leq C h_{T}\left\|\nabla \cdot \mathbf{p}_{h}\right\|_{0, T} .
$$

The bounds (7.1) and (7.2) imply that $\left\|\mathbf{p}_{h}-\widetilde{\mathbf{p}}_{h}\right\|_{\text {div }, \widetilde{T}} \leq C h_{T}\left\|\mathbf{p}_{h}\right\|_{\text {div }, T}$, whence the result follows readily.

Now we are ready to study the discrete approximate sesquilinear forms.

Proposition 7.5. For all $\mathbf{p}_{h} \in X_{h}$ and $u_{h} \in L_{h}$,

$$
b\left(\widetilde{\mathbf{p}}_{h}, \widetilde{u}_{h}\right)=b_{h}\left(\mathbf{p}_{h}, u_{h}\right) .
$$

Proof. Since $\left.\widetilde{u}_{h}\right|_{\widetilde{T}}=\left.u_{h}\right|_{T}=: u_{T}$ is constant, it follows that

$$
\begin{aligned}
\int_{\widetilde{T}} \widetilde{u}_{h}\left(\nabla \cdot \widetilde{\mathbf{p}}_{h}\right)-\int_{T} u_{h}\left(\nabla \cdot \mathbf{p}_{h}\right) & =u_{T}\left(\int_{\widetilde{T}} \nabla \cdot \widetilde{\mathbf{p}}_{h}-\int_{T} \nabla \cdot \mathbf{p}_{h}\right) \\
& =u_{T}\left(\int_{\partial \widetilde{T}} \widetilde{\mathbf{p}}_{h} \cdot \nu-\int_{\partial T} \mathbf{p}_{h} \cdot \nu\right)=0 .
\end{aligned}
$$


The last equality follows from the fact that each integral adds the three degrees of freedom associated to the triangle and that their values coincide.

Proposition 7.6. For all $u_{h}, v_{h} \in L_{h}$,

$$
\left|\left(\rho \widetilde{u}_{h}, \widetilde{v}_{h}\right)-\left(\rho u_{h}, v_{h}\right)_{h}\right| \leq C h\left|u_{h}\right|_{h}\left|v_{h}\right|_{h}
$$

Proof. Let $u_{T}:=\left.u_{h}\right|_{T}$ and $v_{T}:=\left.v_{h}\right|_{T}$. Then,

$$
\left|\int_{\Omega_{-}} \rho \widetilde{u}_{h} \widetilde{v}_{h}-\int_{\Omega_{h}} \rho u_{h} v_{h}\right| \leq \sum_{T \in \mathcal{T}_{h}}\left|u_{T}\right|\left|v_{T}\right|\left|\int_{\widetilde{T}} \rho-\int_{T} \rho\right| .
$$

Since

we finally deduce that

$$
\left|\int_{\widetilde{T}} \rho-\int_{T} \rho\right| \leq C \operatorname{area}(\widetilde{T} \backslash T \cup T \backslash \widetilde{T}) \leq C^{\prime} h_{T}^{3},
$$

$$
\left|\left(\rho \widetilde{u}_{h}, \widetilde{v}_{h}\right)-\left(\rho u_{h}, v_{h}\right)_{h}\right| \leq C h \sum_{T \in \mathcal{T}_{h}} h_{T}^{2}\left|u_{T}\right|\left|v_{T}\right| \leq C^{\prime} h\left|u_{h}\right|_{h}\left|v_{h}\right|_{h},
$$

applying that $C_{1} h_{T}^{2} \leq \operatorname{area}(T) \leq C_{2} h_{T}^{2}$ with $C_{1}, C_{2}$ independent of $T$.

Lemma 7.7. Let $U_{T}:=\left(1 /\left|\widetilde{J}_{T}\right|\right) \widetilde{B}_{T}^{\top} \widetilde{B}_{T}-\left(1 /\left|J_{T}\right|\right) B_{T}^{\top} B_{T}$. Then, there exists $C>0$, independent of $T$, such that $\left\|U_{T}\right\|_{\infty, \widehat{T}} \leq C h_{T}$.

Proof. It is clear that $U_{T}=\left(1 /\left|\widetilde{J}_{T}\right|-1 /\left|J_{T}\right|\right) \widetilde{B}_{T}^{\top} \widetilde{B}_{T}+\left(1 /\left|J_{T}\right|\right)\left(\widetilde{B}_{T}^{\top} \widetilde{B}_{T}-B_{T}^{\top} B_{T}\right)$. On the one hand

$$
1 /\left|\widetilde{J}_{T}\right|-1 /\left|J_{T}\right|=\left(1 /\left|\widetilde{J}_{T}\right|\right)\left(1-\left|\operatorname{det}\left(B_{T}^{-1} \widetilde{B}_{T}\right)\right|\right)=\left(1 /\left|\widetilde{J}_{T}\right|\right)\left(1-\left|\operatorname{det}\left(I+B_{T}^{-1} \Theta_{T}\right)\right|\right)
$$

and, on the other hand, $\widetilde{B}_{T}^{\top} \widetilde{B}_{T}-B_{T}^{\top} B_{T}=\Theta_{T}^{\top} B_{T}+B_{T}^{\top} \Theta_{T}+\Theta_{T}^{\top} \Theta_{T}$. Therefore, the result follows from Proposition 6.1.

Proposition 7.8. For all $\mathbf{p}_{h}, \mathbf{q}_{h} \in X_{h}$,

$$
\left|a\left(\widetilde{\mathbf{p}}_{h}, \widetilde{\mathbf{q}}_{h}\right)-a_{h}\left(\mathbf{p}_{h}, \mathbf{q}_{h}\right)\right| \leq C h\left\|\mathbf{p}_{h}\right\|_{\operatorname{div}, h}\left\|\mathbf{q}_{h}\right\|_{\operatorname{div}, h} .
$$

Proof. Let $\mu:=1 / \sigma$ and $\mu_{T}$ be the value of $\mu$ in the barycenter of $T$ (or its mean value over $T$ ). Then,

$$
\begin{aligned}
\int_{\widetilde{T}} \mu \widetilde{\mathbf{p}}_{h} \cdot \widetilde{\mathbf{q}}_{h}-\int_{T} \mu \mathbf{p}_{h} \cdot \mathbf{q}_{h}= & \int_{\widetilde{T}}\left(\mu-\mu_{T}\right) \widetilde{\mathbf{p}}_{h} \cdot \widetilde{\mathbf{q}}_{h}+\int_{T}\left(\mu_{T}-\mu\right) \mathbf{p}_{h} \cdot \mathbf{q}_{h} \\
& +\mu_{T}\left[\int_{\widetilde{T}} \widetilde{\mathbf{p}}_{h} \cdot \widetilde{\mathbf{q}}_{h}-\int_{T} \mathbf{p}_{h} \cdot \mathbf{q}_{h}\right] .
\end{aligned}
$$

It is clear that

$$
\left|\int_{\widetilde{T}}\left(\mu-\mu_{T}\right) \widetilde{\mathbf{p}}_{h} \cdot \widetilde{\mathbf{q}}_{h}\right| \leq C h_{T}\left\|\widetilde{\mathbf{p}}_{h}\right\|_{\operatorname{div}, \widetilde{T}}\left\|\widetilde{\mathbf{q}}_{h}\right\|_{\operatorname{div}, \widetilde{T}} \leq C^{\prime} h_{T}\left\|\mathbf{p}_{h}\right\|_{\operatorname{div}, T}\left\|\mathbf{q}_{h}\right\|_{\operatorname{div}, T}
$$

where we have applied the bounds (6.7) for the last inequality, and that (recall the assumptions on $\sigma$ in the Remark at Sect. 3),

$$
\left|\int_{T}\left(\mu_{T}-\mu\right) \mathbf{p}_{h} \cdot \mathbf{q}_{h}\right| \leq C h_{T}\left\|\mathbf{p}_{h}\right\|_{\operatorname{div}, T}\left\|\mathbf{q}_{h}\right\|_{\operatorname{div}, T}
$$


For arbitrary $T$, let again $\widehat{\mathbf{p}}_{T}$ be taken so that

$$
\left.\widetilde{\mathbf{p}}_{h}\right|_{\widetilde{T}}=\widetilde{\mathcal{P}}_{T} \widehat{\mathbf{p}}_{T},\left.\quad \mathbf{p}_{h}\right|_{T}=\mathcal{P}_{T} \widehat{\mathbf{p}}_{T},
$$

and let $\widehat{\mathbf{q}}_{T}$ be defined likewise. Hence,

$$
\int_{\widetilde{T}} \widetilde{\mathbf{p}}_{h} \cdot \widetilde{\mathbf{q}}_{h}=\int_{\widehat{T}}\left|\widetilde{J}_{T}\right|\left(\widetilde{\mathbf{p}}_{h} \circ \widetilde{F}_{T}\right) \cdot\left(\widetilde{\mathbf{q}}_{h} \circ \widetilde{F}_{T}\right)=\int_{\widehat{T}}\left(1 /\left|\widetilde{J}_{T}\right|\right)\left(\widetilde{B}_{T} \widehat{\mathbf{p}}_{T}\right) \cdot\left(\widetilde{B}_{T} \widehat{\mathbf{q}}_{T}\right)
$$

and thus

$$
\int_{\widetilde{T}} \widetilde{\mathbf{p}}_{h} \cdot \widetilde{\mathbf{q}}_{h}-\int_{T} \mathbf{p}_{h} \cdot \mathbf{q}_{h}=\int_{\widehat{T}} U_{T} \widehat{\mathbf{p}}_{T} \cdot \widehat{\mathbf{q}}_{T},
$$

$U_{T}$ being the matrix-valued function of Lemma 7.7. Applying this Lemma and bound (6.3) it follows that

$$
\left|\int_{\widetilde{T}} \widetilde{\mathbf{p}}_{h} \cdot \widetilde{\mathbf{q}}_{h}-\int_{T} \mathbf{p}_{h} \cdot \mathbf{q}_{h}\right| \leq\left\|U_{T}\right\|_{\infty, \widehat{T}}\left\|\widehat{\mathbf{p}}_{T}\right\|_{0, \widehat{T}}\left\|\widehat{\mathbf{q}}_{T}\right\|_{0, \widehat{T}} \leq C h_{T}\left\|\mathbf{p}_{h}\right\|_{0, T}\left\|\mathbf{q}_{h}\right\|_{0, T}
$$

Finally,

$$
\left|a\left(\widetilde{\mathbf{p}}_{h}, \widetilde{\mathbf{q}}_{h}\right)-a_{h}\left(\mathbf{p}_{h}, \mathbf{q}_{h}\right)\right| \leq \sum_{T \in \mathcal{T}_{h}} C h_{T}\left\|\mathbf{p}_{h}\right\|_{\operatorname{div}, T}\left\|\mathbf{q}_{h}\right\|_{\operatorname{div}, T} \leq C h\left\|\mathbf{p}_{h}\right\|_{\operatorname{div}, h}\left\|\mathbf{q}_{h}\right\|_{\operatorname{div}, h},
$$

which concludes the proof.

\section{AnAlysis OF THE METHOD}

Here we prove a uniform inf-sup condition associated to the discrete scheme applied to the operator $\mathcal{A}$. It can be understood as a stability condition and ensures existence and uniqueness of solution to problem (3.1). In Section 5 we have already shown a stability result for the same method applied to $\mathcal{A}_{0}$. Stability and convergence analysis will be performed by comparison with the Galerkin method (6.10), applying the results of Section 4 .

We will use again the notation $\mathcal{V}:=H\left(\operatorname{div}, \Omega_{-}\right) \times L^{2}\left(\Omega_{-}\right) \times H^{1 / 2} \times H^{-1 / 2}$ and write $\mathcal{V}_{h, H}:=X_{h} \times L_{h} \times S_{H} \times T_{H}$ for the discrete product space, $\|\cdot\|$ and $\|\cdot\|_{h, H}$ being their respective norms. The brackets $\langle\cdot, \cdot\rangle_{h, H}$ are used to group the duality brackets in the components of $\mathcal{V}_{h, H}$. The discrete counterpart to the operator equation in variational form (2.4) is simply

$$
\mid \begin{aligned}
& \boldsymbol{\varphi}_{h, H}=\left(\mathbf{p}_{h}, u_{h}, \xi_{H}, \psi_{H}\right)^{\top} \in \mathcal{V}_{h, H}, \\
& \left\langle\mathcal{A} \boldsymbol{\varphi}_{h, H}, \boldsymbol{\psi}_{h, H}\right\rangle_{h, H}=\left\langle g, \boldsymbol{\psi}_{h, H}\right\rangle_{h, H}, \quad \forall \boldsymbol{\psi}_{h, H} \in \mathcal{V}_{h, H} .
\end{aligned}
$$

Defining $\widetilde{\mathcal{V}}_{h, H}:=\widetilde{X}_{h} \times \widetilde{L}_{h} \times \widetilde{S}_{H} \times \widetilde{T}_{H}$, the auxiliary BEM-FEM method introduced in (6.10) can be written as

$$
\mid \begin{aligned}
& \widetilde{\boldsymbol{\varphi}}_{h, H}=\left(\widetilde{\mathbf{p}}_{h}, \widetilde{u}_{h}, \widetilde{\xi}_{H}, \widetilde{\psi}_{H}\right)^{\top} \in \widetilde{\mathcal{V}}_{h, H}, \\
& \left\langle\mathcal{A} \widetilde{\boldsymbol{\varphi}}_{h, H}, \widetilde{\boldsymbol{\psi}}_{h, H}\right\rangle=\left\langle g, \widetilde{\boldsymbol{\psi}}_{h, H}\right\rangle, \quad \forall \widetilde{\boldsymbol{\psi}}_{h, H} \in \widetilde{\mathcal{V}}_{h, H} .
\end{aligned}
$$

As a straightforward application of Propositions 7.1, 7.5, 7.6 and 7.8 we derive the existence of $C>0$, independent of $h$ and $H$, such that for all $\varphi_{h, H}, \boldsymbol{\psi}_{h, H} \in \mathcal{V}_{h, H}$,

$$
\begin{aligned}
\left|\left\langle\mathcal{A}_{0} \boldsymbol{\varphi}_{h, H}, \boldsymbol{\psi}_{h, H}\right\rangle_{h, H}-\left\langle\mathcal{A}_{0} \Theta_{h, H} \boldsymbol{\varphi}_{h, H}, \Theta_{h, H} \boldsymbol{\psi}_{h, H}\right\rangle\right| & \leq C h\left\|\boldsymbol{\varphi}_{h, H}\right\|_{h, H}\left\|\boldsymbol{\psi}_{h, H}\right\|_{h, H}, \\
\left|\left\langle\mathcal{A} \boldsymbol{\varphi}_{h, H}, \boldsymbol{\psi}_{h, H}\right\rangle_{h, H}-\left\langle\mathcal{A} \Theta_{h, H} \boldsymbol{\varphi}_{h, H}, \Theta_{h, H} \boldsymbol{\psi}_{h, H}\right\rangle\right| & \leq C h\left\|\boldsymbol{\varphi}_{h, H}\right\|_{h, H}\left\|\boldsymbol{\psi}_{h, H}\right\|_{h, H} .
\end{aligned}
$$


Theorem 8.1. There exists $C>0$, such that for all $h$ and $H$ small enough

$$
\sup _{\boldsymbol{\psi}_{h, H} \in \mathcal{V}_{h, H}} \frac{\left|\left\langle\mathcal{A} \boldsymbol{\varphi}_{h, H}, \boldsymbol{\psi}_{h, H}\right\rangle_{h, H}\right|}{\left\|\boldsymbol{\psi}_{h, H}\right\|_{h, H}} \geq C\left\|\boldsymbol{\varphi}_{h, H}\right\|_{h, H}, \quad \forall \boldsymbol{\varphi}_{h, H} \in \mathcal{V}_{h, H}
$$

In particular, problem (8.1) has a unique solution. Moreover, if $\varphi_{h, H}$ is the solution to problem (8.1), then

$$
\left\|\boldsymbol{\varphi}_{h, H}\right\|_{h, H} \leq C\left(\left\|g_{0}\right\|_{1 / 2}+\left\|g_{1}\right\|_{-1 / 2}\right)
$$

Proof. By bound (8.3) and Proposition 5.3, we can apply Theorem 4.1 to deduce that

$$
\sup _{\widetilde{\boldsymbol{\psi}}_{h, H} \in \widetilde{\mathcal{V}}_{h, H}} \frac{\left|\left\langle\mathcal{A}_{0} \widetilde{\boldsymbol{\varphi}}_{h, H}, \widetilde{\boldsymbol{\psi}}_{h, H}\right\rangle\right|}{\left\|\widetilde{\boldsymbol{\psi}}_{h, H}\right\|} \geq C\left\|\widetilde{\boldsymbol{\varphi}}_{h, H}\right\|, \quad \forall \widetilde{\boldsymbol{\varphi}}_{h, H} \in \widetilde{\mathcal{V}}_{h, H}
$$

Moreover, since $\mathcal{A}-\mathcal{A}_{0}$ is compact and the discrete spaces $\widetilde{\mathcal{V}}_{h, H}$ satisfy the approximation property in $\mathcal{V}$ for $(h, H) \rightarrow(0,0)$, it follows that

$$
\sup _{\tilde{\boldsymbol{\psi}}_{h, H} \in \widetilde{\mathcal{V}}_{h, H}} \frac{\left|\left\langle\mathcal{A} \widetilde{\boldsymbol{\varphi}}_{h, H}, \widetilde{\boldsymbol{\psi}}_{h, H}\right\rangle\right|}{\left\|\widetilde{\boldsymbol{\psi}}_{h, H}\right\|} \geq C\left\|\widetilde{\boldsymbol{\varphi}}_{h, H}\right\|, \quad \forall \widetilde{\boldsymbol{\varphi}}_{h, H} \in \widetilde{\mathcal{V}}_{h, H}
$$

for $(h, H)$ small enough, since convergence of Galerkin methods is preserved by compact perturbations (see [22], Thm. 13.7). To show the inf-sup condition (8.5) we can apply again Theorem 4.1 taking into account that conditions (8.4) and (8.7) are satisfied. The last assertion follows readily now from condition (8.5) since $g=\left(0,0, g_{1},-g_{0}\right)^{\top}$. Therefore, if $\boldsymbol{\varphi}_{h, H} \in \mathcal{V}_{h, H}$ is the solution to problem (8.1), then,

$$
\left\|\boldsymbol{\varphi}_{h, H}\right\|_{h, H} \leq C \sup _{\boldsymbol{\psi}_{h, H} \in \mathcal{V}_{h, H}} \frac{\left|\left\langle g, \boldsymbol{\psi}_{h, H}\right\rangle_{h, H}\right|}{\left\|\boldsymbol{\psi}_{h, H}\right\|_{h, H}} \leq C\left(\left\|g_{0}\right\|_{1 / 2}+\left\|g_{1}\right\|_{-1 / 2}\right) .
$$

Theorem 8.2. There exists $C>0$, independent of $h$ and $H$, such that

$$
\left\|\boldsymbol{\varphi}-\boldsymbol{\varphi}_{h, H}\right\| \leq C\left(\inf _{\widetilde{\boldsymbol{\psi}}_{h, H} \in \widetilde{\mathcal{V}}_{h, H}}\left\|\boldsymbol{\varphi}-\widetilde{\boldsymbol{\psi}}_{h, H}\right\|+h\|\boldsymbol{\varphi}\|\right),
$$

$\boldsymbol{\varphi}$ and $\boldsymbol{\varphi}_{h, H}$ being the solutions to problems (2.4) and (8.1) respectively. If $\boldsymbol{\varphi}=(\mathbf{p}, u, \xi, \psi) \in H^{1}\left(\operatorname{div}, \Omega_{-}\right) \times$ $H^{1}\left(\Omega_{-}\right) \times H^{3 / 2} \times H^{1 / 2}$, then

$$
\begin{aligned}
\left\|\mathbf{p}-\mathbf{p}_{h}\right\|_{\text {div }}+\left\|u-u_{h}\right\|_{0}+\left\|\xi-\xi_{H}\right\|_{1 / 2}+\left\|\psi-\psi_{H}\right\|_{-1 / 2} \\
\leq C(h+H)\left(\|\mathbf{p}\|_{1, \text { div }}+\|u\|_{1, \Omega_{-}}+\|\xi\|_{3 / 2}+\|\psi\|_{1 / 2}\right) .
\end{aligned}
$$

Proof. Since the uniform bound (8.4) and the inf-sup condition in Theorem 8.1 hold, applying Theorem 4.2 we deduce that

$$
\left\|\boldsymbol{\varphi}-\Theta_{h, H} \boldsymbol{\varphi}_{h, H}\right\| \leq C\left(\inf _{\widetilde{\boldsymbol{\psi}}_{h, H} \in \widetilde{\mathcal{V}}_{h, H}}\left\|\boldsymbol{\varphi}-\widetilde{\boldsymbol{\psi}}_{h, H}\right\|+h\|\boldsymbol{\varphi}\|\right)
$$

Notice that as a consequence of Proposition 7.4 and the fact that $\widetilde{u}_{h}=u_{h}$ in $\Omega_{-} \cap \Omega_{h}$, it follows that

$$
\left\|\Theta_{h, H} \boldsymbol{\varphi}_{h, H}-\boldsymbol{\varphi}_{h, H}\right\| \leq C h\left\|\boldsymbol{\varphi}_{h, H}\right\|_{h, H}, \quad \forall \boldsymbol{\varphi}_{h, H} \in \mathcal{V}_{h, H},
$$


where $\mathbf{p}_{h}$ and $u_{h}$ are extended to $\Omega_{-} \backslash \Omega_{h}$ by their corresponding local expressions in the nearest $T$. Using the estimate (8.6), the result follows readily.

The solution to the original transmission problem (1.2) in the exterior domain is given as a single layer potential. We can then obtain pointwise error estimates in $\Omega_{+}$.

Theorem 8.3. If $\varphi \in H^{1}\left(\operatorname{div}, \Omega_{-}\right) \times H^{1}\left(\Omega_{-}\right) \times H^{3 / 2} \times H^{1 / 2}$, then

$$
\left|\mathcal{S}_{\lambda} \psi(\mathbf{z})-\mathcal{S}_{\lambda} \psi_{H}(\mathbf{z})\right|=\mathcal{O}_{\mathbf{z}}(h+H), \quad \mathbf{z} \in \Omega_{+}
$$

Proof. It follows from the fact that

$$
\left|\mathcal{S}_{\lambda}\left(\psi-\psi_{H}\right)(\mathbf{z})\right|=\left|\left\langle\psi-\psi_{H}, \frac{\imath}{4} H_{0}^{(1)}(\lambda|\mathbf{z}-\mathbf{x}(\cdot)|)\right\rangle\right| \leq C_{\mathbf{z}}\left\|\psi-\psi_{H}\right\|_{-1 / 2} .
$$

Applying Theorem 8.2, we conclude the proof.

Even though the constant $C_{\mathbf{z}}$ in Theorem 8.3 depends on $\mathbf{z}$, it is uniformly bounded in the exterior of any ball enclosing $\Gamma$, and only blows up when we approach $\Gamma$. We can also obtain a bound of the same order for $\left\|\mathcal{S}_{\lambda} \psi-\mathcal{S}_{\lambda} \psi_{H}\right\|_{1, \Omega_{+}}$. The bound of Theorem 8.3 could be improved if we had higher order bounds for the approximation of $\psi-\psi_{H}$ in weaker norms. These ones, in its turn, would follow from Aubin-Nitsche type estimates, although how to apply them to this situation is right now out of our reach.

\section{Numerical Results AND CONCLUSions}

It is possible to construct a fully discrete version of the method (8.1) by using elementary ideas of numerical quadrature. We do not give here a detailed description and analysis of the method for the sake of brevity. The analysis can be done by applying similar results to Theorems 4.1 and 4.2 and following basically the same ideas as in $[9,40]$, with suitable modifications and new technicalities. This is done in [38].

To approximate the integrals related to the operator $V_{\lambda}$ we use a generalization of the Galerkin-collocation method (see $[9,18,19])$ based on a subtraction of the logarithmic singularity, an exact computation of the singular part, and a simple midpoint quadrature rule for the remaining term. For the right hand side in scheme (8.1) as well as for the integrals related to the operator $J_{\lambda}$ we use midpoint rules. This is one of the methods described in [39]. The integrals over triangles of $\mathcal{T}_{h}$ are approximated with standard quadrature rules.

It can be shown that if $\left(\widehat{\mathbf{p}}_{h}, \widehat{u}_{h}, \widehat{\xi}_{H}, \widehat{\psi}_{H}\right) \in \mathcal{V}_{h, H}$ is the approximate solution to problem (2.4) obtained by the fully discrete method indicated above, and the solution to problem $(2.4)$ belongs to $H^{1}\left(\operatorname{div}, \Omega_{-}\right) \times H^{1}\left(\Omega_{-}\right) \times$ $H^{3 / 2} \times H^{1 / 2}$, then

$$
\left\|\mathbf{p}-\widehat{\mathbf{p}}_{h}\right\|_{\operatorname{div}}+\left\|u-\widehat{u}_{h}\right\|_{0}+\left\|\xi-\widehat{\xi}_{H}\right\|_{1 / 2}+\left\|\psi-\widehat{\psi}_{H}\right\|_{-1 / 2}=\mathcal{O}(h+H) .
$$

Therefore the method has the same convergence order as the method without numerical integration.

Once $\widehat{\psi}_{H}=\sum_{j=1}^{N} \widehat{\psi}_{j} \chi_{j}$ is computed, (here $\chi_{j}$ is the 1-periodized characteristic function of the interval $\left.\left[t_{j}, t_{j+1}\right]\right)$, we can construct a pointwise approximation of the solution to problem (1.2) in $\Omega_{+}$as a fully discrete version of the single layer potential with density $\widehat{\psi}_{H}$ by

$$
\widehat{u}_{h, H}(\mathbf{z}):=\frac{\imath}{4 N} \sum_{j=1}^{N} H_{0}^{(1)}\left(\lambda\left|\mathbf{z}-\mathbf{x}\left(s_{j}\right)\right|\right) \widehat{\psi}_{j}, \quad \mathbf{z} \in \Omega_{+} .
$$

Then, it can be proven that $\left|u(\mathbf{z})-\widehat{u}_{h, H}(\mathbf{z})\right|=\mathcal{O}_{\mathbf{z}}(h+H)$ for $\mathbf{z} \in \Omega_{+}$. Again, pointwise convergence properties are preserved. 


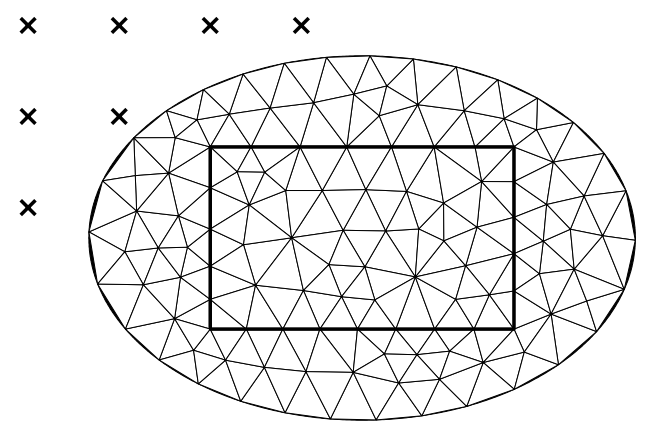

FiguRE 1. Geometry and basic triangulation of the problem.

TABLE 1. Pointwise errors and estimated convergence rates.

\begin{tabular}{|c|c|c|cc|cc|}
\hline$\sharp \mathcal{I}_{h}$ & $n$ & $N$ & $E_{\text {ext }}$ & e.c.r. & $E_{\text {int }}$ & e.c.r. \\
\hline 217 & 31 & 40 & $2.36(-3)$ & & $1.01(-2)$ & \\
868 & 62 & 80 & $6.82(-4)$ & 1.790 & $5.08(-3)$ & 0.994 \\
3472 & 124 & 160 & $1.97(-4)$ & 1.789 & $2.48(-3)$ & 1.033 \\
13888 & 248 & 320 & $4.91(-5)$ & 2.007 & $1.11(-3)$ & 1.160 \\
\hline
\end{tabular}

Now we present here two different numerical examples to illustrate how our method works. In both tests the exact solutions are not known, so we have computed the approximated solution on several refined meshes in order to estimate the order of convergence. As the exact solution to compare with, we take one computed with the same method but using the finest triangulation of the successive refinements (with 55552 triangles in the first experiment and 45568 in the second one) and with a completely different discretization of the boundary integral equations with a significantly higher number of nodes (1000 in both examples).

Locally non-homogeneous media with piecewise constant properties. In this first numerical illustration we solve problem (1.2) when $\Gamma$ is the ellipse centered at $(0,0)$ with semiaxes 0.45 and 0.3 . For the material occupying the exterior domain we take the constant values $\rho=1$ and $\sigma=1$. The interior material is filled with two homogeneous materials with different properties. The kernel (of rectangular shape, see Fig. 1) is occupied by a material with thermal constants $\rho=1$ and $\sigma=1 / 300$. With these values, the kernel is a worse heat conductor than the matrix. The remaining annular region is filled with a better (comparing with the matrix) heat conducting material with thermal constants $\rho=1$ and $\sigma=30$. Although $\sigma$ is not even continuous, we choose triangulations that are adapted to its regularity (see Fig. 1 where the initial triangulation is represented). Therefore, numerical integration does not affect convergence.

We compute the sum of the errors in the seven exterior points represented in Figure 1,

$$
E_{\mathrm{ext}}:=\sum_{i=1}^{7}\left|u\left(x_{i}\right)-\widehat{u}_{h, H}\left(x_{i}\right)\right| .
$$

The exact solution in these points satisfies $\sum_{i=1}^{7}\left|u\left(x_{i}\right)\right| \approx 0.8139$. In the interior domain we compute the $L^{2}$ norm, $E_{\text {int }}:=\left\|u-\widehat{u}_{h, H}\right\|_{L^{2}\left(\Omega_{h}\right)}$. For the exact solution we have that $\|u\|_{L^{2}\left(\Omega_{h}\right)} \approx 0.6097$. We write in Table 1 the errors $E_{\text {ext }}$ and $E_{\text {int }}$ as well as the estimated convergence rates (e.c.r.) computed by comparing the errors on consecutive grids in a standard way.

Numerical experiments show that the number of degrees of freedom for the boundary approximation $(N)$ and the number of sides of triangles on the boundary stemming from the triangulation $(n)$ have to be almost 
TABLE 2. Pointwise errors and estimated convergence rates.

\begin{tabular}{|c|c|c|cc|cc|}
\hline$\sharp \mathcal{T}_{h}$ & $n$ & $N$ & $E_{\text {ext }}$ & e.c.r. & $E_{\text {int }}$ & e.c.r. \\
\hline 178 & 32 & 45 & $2.96(-3)$ & & $4.25(-3)$ & \\
712 & 64 & 90 & $6.73(-4)$ & 2.137 & $2.21(-3)$ & 1.004 \\
2848 & 128 & 180 & $1.57(-4)$ & 2.094 & $1.03(-3)$ & 1.034 \\
11392 & 256 & 360 & $3.19(-5)$ & 2.303 & $4.63(-4)$ & 1.160 \\
\hline
\end{tabular}

equal to optimize efforts. That is, increasing $N$ for fixed $n$ or vice versa does not provide better approximations than for $N$ and $n$ almost equal.

Notice that the discretization seems to have quadratic order in the exterior domain. We point out that if we deal with locally-homogeneous media, that is, with constant coefficients in both domains, and solve the problem using a boundary indirect formulation looking for the solution in the form of a single layer potential in each domain, and for the approximation of the equivalent system of integral equations we use a Petrov-Galerkin scheme with $S_{H} \times S_{H}$ and $S_{H} \times T_{H}$ as trial and test spaces respectively, then, in weak norms, the approximation of the densities has quadratic order (see $[38,39]$ ). The corresponding fully discrete method with the same approximations as those we perform here has order two both for densities in weak norms and pointwise approximation.

Locally non-homogeneous media. In this example the geometry of the problem is the same as before. The material occupying the ellipse has now non-constant properties:

$$
\rho(x, y):=50(x-0.5)(y-0.5)+0.01, \quad \sigma(x, y):=x^{2}+y^{2}+0.5, \quad \forall(x, y) \in \Omega_{-} .
$$

For the exterior domain we simply take $\rho=1$ and $\sigma=1$. The exact solution satisfies that $\sum_{i=1}^{7}\left|u\left(x_{i}\right)\right| \approx 2.7176$ and $\|u\|_{L^{2}\left(\Omega_{h}\right)} \approx 0.3996$. The numerical results are given in Table 2 and show the same behaviour as before.

Final comments. The method we have presented and analysed in the preceding pages provides a simple way of obtaining numerical simulations for the problem of scattering of thermal waves in some situations of practical interest. An advantage of the method is the fact that the FEM and BEM routines work almost independently with a small coupling term. Its simplicity makes it attractive for the repeated computations needed to deal with inverse or parameter determination problems, typical of the realm of interest of thermal waves. We point out here some possible extensions, which will be the object of future work.

The BEM discretization we have used can be substituted by any other one with the same stability properties. Some examples of pairs of spaces are provided in [39]. The method chosen here is the lowest order pair. The choice was driven from the aim of keeping the method as simple as possible. We also wanted to avoid a complicated BEM routine since the mixed FEM block has only order one. The strategy developed in $[27,31]$ offers a different point of view: the use of a spectral approximation of the boundary integral equation allows to think of that discretization as an approximation of the exact absorbing boundary condition in the exterior boundary and concentrate the remaining computational effort in the interior domain.

The extension to three dimensions is a priori simple. As we have already mentioned in Section 2, the wellposedness of the four-field formulation holds in this case. The choice of adequate spaces for the separate FEM and BEM codes is also feasible, but two difficulties arise now. The first one, of a theoretical character, is the analysis of the method by comparison with a curved Galerkin mixed method. We believe that extensions of the two-dimensional bounds of Section 6 are possible ([24] gives many bounds in this line). The practical difficulty arises from the non-matching grids of the FEM and BEM routines, that can cause serious complications in coding the method in an efficient way. Finally, there is an additional possibility of extending the analysis to non-linear (Lipschitz strongly monotone) problems, which has to be examined in detail in the future. 
Acknowledgements. The authors are partially supported by MEC/FEDER Project MTM2004-01905, Gobierno de Aragón (Grupo Consolidado PDIE) and by Gobierno de Navarra Ref. 18/2005.

\section{REFERENCES}

[1] D.P. Almond and P.M. Patel, Photothermal science and techniques. Chapman and Hall, London (1996).

[2] J.-P. Aubin, Approximation of elliptic boundary-value problems. Wiley-Interscience, New York-London-Sydney (1972).

[3] H.T. Banks, F. Kojima and W.P. Winfree, Boundary estimation problems arising in thermal tomography. Inverse Problems 6 (1990) 897-921.

[4] F. Brezzi and M. Fortin, Mixed and hybrid finite element methods. Springer-Verlag, New York (1991).

[5] F. Brezzi and C. Johnson, On the coupling of boundary integral and finite element methods. Calcolo 16 (1979) $189-201$.

[6] G. Chen and J. Zhou, Boundary element methods. Academic Press, London (1992).

[7] M. Costabel, Symmetric methods for the coupling of finite elements and boundary elements. Boundary elements IX, Vol. 1 (Stuttgart, 1987), Comput. Mech. (1987) 411-420.

[8] M. Costabel and E. Stephan, A direct boundary integral equation method for transmission problems. J. Math. Anal. Appl. 106 (1985) 367-413.

[9] M. Crouzeix and F.-J. Sayas, Asymptotic expansions of the error of spline Galerkin boundary element methods. Numer. Math. 78 (1998) 523-547.

[10] F. Garrido and A. Salazar, Thermal wave scattering by spheres. J. Appl. Phys. 95 (2004) 140-149.

[11] G.N. Gatica and G.C. Hsiao, On the coupled BEM and FEM for a nonlinear exterior Dirichlet problem in $\mathbb{R}^{2}$. Numer. Math. 61 (1992) 171-214.

[12] G.N. Gatica and G.C. Hsiao, Boundary-field equation methods for a class of nonlinear problems. Pitman Research Notes in Mathematics Series 331, Longman Scientific and Technical, Harlow, UK (1995).

[13] G.N. Gatica and S. Meddahi, A dual-dual mixed formulation for nonlinear exterior transmission problems. Math. Comp. 70 (2001) 1461-1480.

[14] V. Girault and P.-A. Raviart, Finite element methods for Navier-Stokes equations. Theory and algorithms. Springer-Verlag, New York (1986).

[15] H. Han, A new class of variational formulations for the coupling of finite and boundary element methods. J. Comput. Math. 8 (1990) 223-232.

[16] T. Hohage, M.-L. Rapún and F.-J. Sayas, Detecting corrosion using thermal measurements. Inverse Probl. (to appear).

[17] G.C. Hsiao, The coupling of BEM and FEM - a brief review. Boundary elements X, Vol 1 (Southampton, 1988). Comput. Mech. (1988) 431-445.

[18] G.C. Hsiao, P. Kopp and W.L. Wendland, A Galerkin collocation method for some integral equations of the first kind. Computing 25 (1980) 89-130.

[19] G.C. Hsiao, P. Kopp and W.L. Wendland, Some applications of a Galerkin-collocation method for boundary integral equations of the first kind. Math. Method. Appl. Sci. 6 (1984) 280-325.

[20] C. Johnson and J.-C. Nédélec, On the coupling of boundary integral and finite element methods. Math. Comp. 35 (1980) 1063-1079

[21] R.E. Kleinman and P.A. Martin, On single integral equations for the transmission problem of acoustics. SIAM J. Appl. Math 48 (1988) 307-325.

[22] R. Kress, Linear integral equations. Second edition. Springer-Verlag, New York (1999).

[23] R. Kress and G.F. Roach, Transmission problems for the Helmholtz equation. J. Math. Phys. 19 (1978) $1433-1437$.

[24] M. Lenoir, Optimal isoparametric finite elements and error estimates for domains involving curved boundaries. SIAM J. Num Anal. 23 (1986) 562-580.

[25] A. Mandelis, Photoacoustic and thermal wave phenomena in semiconductors. North-Holland, New York (1987).

[26] A. Mandelis, Diffusion-wave fields. Mathematical methods and Green functions. Springer-Verlag, New York (2001).

[27] A. Márquez, S. Meddahi and V. Selgas, A new BEM-FEM coupling strategy for two-dimensional fluid-solid interaction problems. J. Comput. Phys. 199 (2004) 205-220.

[28] W. McLean, Strongly elliptic systems and boundary integral equations. Cambridge University Press, Cambridge (2000).

[29] S. Meddahi, A mixed-FEM and BEM coupling for a two-dimensional eddy current problem. Numer. Funct. Anal. Optim. 22 (2001) 127-141.

[30] S. Meddahi and A. Márquez, A combination of spectral and finite elements for an exterior problem in the plane. Appl. Numer. Math. 43 (2002) 275-295.

[31] S. Meddahi and F.-J. Sayas, A fully discrete BEM-FEM for the exterior Stokes problem in the plane. SIAM J. Numer. Anal. 37 (2000) 2082-2102.

[32] S. Meddahi and F.-J. Sayas, Analysis of a new BEM-FEM coupling for two-dimensional fluid-solid interaction. Numer. Methods Partial Differ. Equ. 21 (2005) 1017-1042.

[33] S. Meddahi and V. Selgas, A mixed-FEM and BEM coupling for a three-dimensional eddy current problem. ESAIM: M2AN 37 (2003) 291-318. 
[34] S. Meddahi, J. Valdés, O. Menéndez and P. Pérez, On the coupling of boundary integral and mixed finite element methods. J. Comput. Appl. Math. 69 (1996) 113-124.

[35] S. Meddahi, A. Márquez and V. Selgas, Computing acoustic waves in an inhomogeneous medium of the plane by a coupling of spectral and finite elements. SIAM J. Numer. Anal. 41 (2003) 1729-1750.

[36] S.G. Mikhlin, Mathematical Physics, an advanced course. North-Holland, Amsterdam-London (1970).

[37] L. Nicolaides and A. Mandelis, Image-enhanced thermal-wave slice diffraction tomography with numerically simulated reconstructions. Inverse problems 13 (1997) 1393-1412.

[38] M.-L. Rapún, Numerical methods for the study of the scattering of thermal waves. Ph.D. Thesis, University of Zaragoza, (2004). In Spanish.

[39] M.-L. Rapún and F.-J. Sayas, Boundary integral approximation of a heat diffusion problem in time-harmonic regime. Numer. Algorithms 41 (2006) 127-160.

[40] F.-J. Sayas, A nodal coupling of finite and boundary elements. Numer. Methods Partial Differ. Equ. 19 (2003) 555-570.

[41] J.M. Terrón, A. Salazar and A. Sánchez-Lavega, General solution for the thermal wave scattering in fiber composites. J. Appl. Phys. 91 (2002) 1087-1098.

[42] R.H. Torres and G.V. Welland, The Helmholtz equation and transmission problems with Lipschitz interfaces. Indiana Univ. Math. J. 42 (1993) 1457-1485.

[43] T. von Petersdorff, Boundary integral equations for mixed Dirichlet, Neumann and transmission problems. Math. Methods Appl. Sci. 11 (1989) 185-213.

[44] A. Ženišek, Nonlinear elliptic and evolution problems and their finite element approximations. Academic Press, London (1990).

[45] M. Zlámal, Curved elements in the finite element method I. SIAM J. Numer. Anal. 10 (1973) 229-240.

[46] M. Zlámal, Curved elements in the finite element method II. SIAM J. Numer. Anal. 11 (1974) 347-362.

To access this journal online:

www.edpsciences.org 